\title{
An XMM-Newton view of the young open cluster NGC 6231
}

\section{The catalogue $e^{\star \star \star \star, \star \star \star}$}

\author{
H. Sana ${ }^{1, \dagger}$, E. Gosset ${ }^{1, \ddagger}$, G. Rauw ${ }^{1, \ddagger}$, H. Sung ${ }^{2}$, and J.-M. Vreux ${ }^{1}$ \\ 1 Institut d'Astrophysique et de Géophysique, University of Liège, Allée du 6 Août 17, Bât. B5c, 4000 Liège, Belgium \\ e-mail: [sana; gosset; rauw; vreux]@astro.ulg.ac.be \\ 2 Department of Astronomy and Space Science, Sejong University, Kunja-dong 98, Kwangjin-gu, Seoul 143-747, Korea \\ e-mail: sungh@sejong.ac.kr
}

Received 11 April 2005 / Accepted 22 March 2006

\begin{abstract}
This paper is the first of a series dedicated to the X-ray properties of the young open cluster NGC 6231. Our data set relies on an XMM-Newton campaign of a nominal duration of $180 \mathrm{ks}$ and reveals that NGC 6231 is very rich in the X-ray domain too. Indeed, $610 \mathrm{X}$-ray sources are detected in the present field of view, centered on the cluster core. The limiting sensitivity of our survey is approximately $6 \times 10^{-15} \mathrm{erg} \mathrm{cm}^{-2} \mathrm{~s}^{-1}$ but clearly depends on the location in the field of view and on the source spectrum. Using different existing catalogues, over $85 \%$ of the X-ray sources could be associated with at least one optical and/or infrared counterpart within a limited cross-correlation radius of $3^{\prime \prime}$ at maximum. The surface density distribution of the X-ray sources presents a slight $\mathrm{N}-\mathrm{S}$ elongation. Once corrected for the spatial sensitivity variation of the EPIC instruments, the radial profile of the source surface density is well described by a King profile with a central density of about 8 sources per $\operatorname{arcmin}^{2}$ and a core radius close to 3.1 arcmin. The distribution of the X-ray sources seems closely related to the optical source distribution. The expected number of foreground and background sources should represent about $9 \%$ of the detected sources, thus strongly suggesting that most of the observed X-ray emitters are physically belonging to NGC 6231. Finally, beside a few bright but soft objects - corresponding to the early-type stars of the cluster - most of the sources are relatively faint $\left(\sim 5 \times 10^{-15} \mathrm{erg} \mathrm{cm}^{-2} \mathrm{~s}^{-1}\right)$ with an energy distribution peaked around 1.0-2.0 keV.
\end{abstract}

Key words. open clusters and associations: individual: NGC 6231 - X-rays: individuals: NGC 6231 - X-rays: stars stars: early-type - catalogues

\section{Introduction}

Detailed studies of young clusters are powerful tools to probe crucial astrophysical issues. Because they a priori contain both early-type stars and pre-main sequence (PMS) stars, young clusters are privileged laboratories to test star formation and evolution theories. They indeed provide a homogeneous sample of stars in terms of distance, reddening, environment, chemical composition and age. With the currently available X-ray observatories, unprecedented investigations of young open clusters in the X-ray domain have been performed in the past few years. The increased sensitivity, spectral power and resolution of the XMM-Newton and Chandra observatories, compared to X-ray satellites of the previous generations, give now a much more

^ Based on observations collected with XMM-Newton, an ESA science mission with instruments and contributions directly funded by ESA Member States and the USA (NASA).

$\star \star$ Full Tables 3 and 6 are only available in electronic form at the CDS via anonymous ftp to

cdsarc.u-strasbg.fr (130.79.128.5) or via

http://cdsweb.u-strasbg.fr/cgi-bin/qcat?]/A+A/454/1047

$\star \star \star$ Appendices are only available in electronic form at

http://www.edpsciences.org

FNRS Research Fellow (Belgium).

FNRS Research Associate (Belgium). complete view of the X-ray properties of the star populations in clusters.

For example, a 76 ks Chandra observation of the embedded young cluster NGC $2024(d \sim 410 \mathrm{pc} ;$ age $=0.3-$ a few Myr $)$ revealed $283 \mathrm{X}$-ray sources displaying heavily absorbed hard spectra with a mean temperature $k T \sim 3 \mathrm{keV}$ (Skinner et al. 2003). A significant fraction (25\%) of the $\mathrm{X}$-ray sources shows a wide range of variability within the exposure duration. In addition, Chandra detected at least $96 \%$ of the known classical T Tauri stars in NGC 2024. Results for other clusters are very similar. Rauw et al. (2003) performed a 20 ks observation of NGC $6383(d \sim 1.4 \mathrm{kpc}$; age $=1.7-5 \mathrm{Myr})$ and found 77 sources, mostly centered on the cluster location. An important fraction of these sources are probable PMS objects. Using both XMM-Newton and Chandra facilities, Preibisch \& Zinnecker (2004, and references therein) studied the very young stellar cluster IC $348(d \sim 310 \mathrm{pc}$; age $\sim 2 \mathrm{Myr})$ and found $286 \mathrm{X}$-ray sources among which over 50 classical T Tauri stars. Comparison of Chandra- and XMM-Newton-based spectral properties suggested that the $\mathrm{X}$-ray characteristics of $\mathrm{T}$ Tauri stars remain mostly constant over periods of years. NGC 6530 $(d \sim 1.8 \mathrm{kpc} ;$ age $\sim 1.5-2.0 \mathrm{Myr})$ is a very rich open cluster containing several massive O-type stars as well as a large population of B-type stars. XMM-Newton observations by Rauw et al. (2002) revealed 119 sources, of which a large fraction are 
PMS candidates. Similarly to Skinner et al. results, the X-ray spectra of the PMS candidates are characterized by temperatures of a few keV. Using a $60 \mathrm{ks}$ Chandra observation centered on the same cluster, Damiani et al. (2004) revealed 884 X-ray sources, among which 90 to $95 \%$ are PMS stars.

From this review of the recent literature, there is an obvious body of observations showing that, besides the expected X-ray emission from massive stars, a large population of X-ray emitting low-mass PMS stars is to be found while observing young clusters. The present study of the very rich cluster NGC 6231 lies in this framework. It aims at a better comprehension of both early-type stars and young open clusters by extending the previous sample of investigations to clusters with a large O-type star population. A severe limitation of several of the above cited works is the lack of detailed studies on the concerned cluster at other wavelengths. Indeed, with Chandra and XMM-Newton, the X-ray observations are so deep that a deep optical photometry of the field is required. Such a data set is indeed essential to, for example, more clearly identify the evolutionary status of the different sub-populations of the cluster. Fortunately, as shown by the literature review of Sect. 2, the stars in NGC 6231 have been thoroughly studied. Together with the depth of the present X-ray campaign, this is one of the strengths of the current work. Finally, the present work distinguishes itself from the previous investigations because of the particular planning of the X-ray observations. Indeed our XMM-Newton campaign towards NGC 6231 was actually split into six successive pointings, spread over a period of five days. This will allow us to probe the variability of the X-ray emission of the detected sources on different time-scales.

A detailed analysis of the central target of the field, the colliding wind binary HD 152248, has been presented recently in a dedicated paper (Sana et al. 2004). The source will therefore not be further discussed in more details in the present paper. Preliminary results from this campaign, mainly related to the early-type X-ray emitters, were also presented in Sana et al. $(2005 b, 2006 b, c)$. In the present paper, we focus on the X-ray catalogue and we discuss some general properties of the detected sources. Other aspects of the X-ray properties of NGC 6231, such as the early-type and the pre-main sequence population characteristics, will be addressed in subsequent papers of this series.

This first paper is organized as follows. After a review of the abundant literature on NGC 6231 and on the Sco OB 1 association, Sect. 3 describes the campaign and the subsequent data reduction processes. In Sect. 4, we address the detection and identification of the sources in the XMM-Newton field of view, and we present the resulting X-ray catalogues. Finally, we probe the main properties of the cluster X-ray emitters (Sect. 5). Section 6 summarizes the results of the present work.

\section{NGC 6231 and the Sco OB 1 association: a literature review}

\subsection{The Sco OB 1 association}

Located in the Sagittarius-Carina spiral arm of our galaxy $\left(\alpha(2000)=16^{\mathrm{h}} 53^{\mathrm{m}} \cdot 6, \delta(2000)=-41^{\circ} 57^{\prime} ; l=343^{\circ} .3, b=1.2\right.$, Perry et al. 1991), the Sco OB 1 association is an extremely rich and interesting region of the sky. $2^{\circ}$ long by $1^{\circ}$ wide, it extends from the gaseous nebula IC 4628 on its northern end to the young open cluster NGC 6231 towards its southern end. Its major axis is approximately parallel to the Galactic plane (Morgan et al. 1953a). A sparser group, $\operatorname{Tr} 24$, is to be found near IC 4628 while two other clusters, NGC 6242 and NGC 6268, lie slightly north of the association. Finally the H II region IC 4878, centered on NGC 6231 , extends by about $4^{\circ}$ by $5^{\circ}$ in the form of an elliptical ring and is probably triggered by the cluster. The emission nebula is faint within the ring but is very bright where the ring is crossed by the northern end of the association (Bok et al. 1966).

The interest in Sco OB 1 mainly originates from its extended early-type star content (Shobbrook 1983; Raboud et al. 1997). Beyond the numerous O- and B-type stars, the association also shelters two of the rare Wolf-Rayet (WR) stars, two Of stars displaying P Cygni profiles as well as several $\beta$ Cephei variables (Balona \& Engelbrecht 1985; Arentoft et al. 2001). Among the peculiar objects found within Sco OB 1 is the bright star $\zeta^{1}$ Sco. With an absolute magnitude around $M_{V}=-8.3, \zeta^{1} \mathrm{Sco}$ is one of the brightest stars of the Milky Way. Many of the "normal" earlytype stars further present signs of variability and have a good chance to be binary systems (e.g. Raboud 1996; Arentoft et al. 2001).

\subsection{The NGC 6231 cluster}

Located near the southern end of the Sco OB 1 association, the young open cluster NGC $6231\left(\alpha(2000)=16^{\mathrm{h}} 54^{\mathrm{m}} 09^{\mathrm{s}}\right.$, $\left.\delta(2000)=-41^{\circ} 49^{\prime} 36^{\prime \prime}\right)$ contains an important number of bright early-type stars in its centre. Often considered as the nucleus of the association (Bok et al. 1966), its relationship to Sco OB 1 has been subject to different interpretations with time. Though Heske \& Wendker (1984) presented evidence that $\operatorname{Tr} 24$ and Sco OB 1 form a single aggregate, these authors proposed that NGC 6231 is actually a foreground cluster. Heske \& Wendker also found a sub-cluster of PMS stars in the vicinity of $\operatorname{Tr} 24$. Based on an extensive set of data, Perry et al. (1990, 1991) re-addressed these issues and carefully studied the interrelation between the three aggregates. They established that Sco OB 1, NGC 6231 and $\operatorname{Tr} 24$ are located at the same distance and have the same age, thus demonstrating that NGC 6231 is not a foreground object but is clearly embedded in the Sco OB 1 association. NGC 6231 therefore retains its status as the nucleus of the association. Perry et al. could however not confirm the three stellar sub-aggregates found by Seggewiss (1968a) in $\operatorname{Tr} 24$ and, as suggested by Heske \& Wendker (1984), they casted further doubts on the physical reality of the Tr 24 aggregate itself. Perry et al. finally confirmed the existence of a PMS sub-cluster near $\operatorname{Tr} 24$.

The properties of NGC 6231 and of its stellar content have been thoroughly investigated during the past century. Three main streams of investigation were indeed designed, namely photometry, spectral classification and radial velocity measurements. The photometric approach is however predominant and was extensively performed using different photometric systems. The bulk of the available literature on the cluster relies on photographic, photoelectric or CCD campaigns: Brownlee \& Cox (1953, PV), Houck (1956), Walraven \& Walraven (1960, Walraven), Feast et al. (1961, $U B V)$, Breckinridge \& Kron (1963, PV), Bok et al. (1966, $U B V \mathrm{H} \beta$ ), Feinstein \& Ferrer (1968, $U B V)$, Seggewiss (1968b, $U B V)$, Schild et al. (1969, $U B V)$, Crawford et al. (1971, uvby $\mathrm{H} \beta)$, Garrison \& Schild (1979, $U B V)$, Shobbrook (1983, uvby $\mathrm{H} \beta$ ), Heske \& Wendker (1984, $U B V)$, van Genderen et al. (1984, Walraven), Perry et al. (1991, uvby), Meynet et al. (1993, UBV), Balona \& Laney (1995, uvby $\mathrm{H} \beta)$, Raboud et al. (1997, Geneva), Sung et al. (1998, $U B V(R I)_{\mathrm{C}} \mathrm{H} \alpha$ ), Baume et al. (1999, $\left.U B V I_{\mathrm{C}}\right)$. The more recent works (from $\sim 1990$ 's) offer a much more complete view of the cluster both in terms of their angular extent and of the 
magnitude limit reached. An extensive still careful comparison of most (if not all) the works published prior to 1990 has been performed by Perry et al. (1991).

Spectral classification of the cluster objects has mainly been carried out by Morgan et al. (1953b), Houck (1956), Feast et al. (1961), Schild et al. (1969), Garrison \& Schild (1979), Levato \& Malaroda (1980), Conti \& Alschuler (1971), Walborn (1972), Mathys (1988, 1989), García \& Mermilliod (2001) and Sana (2005). Radial velocity campaigns were led essentially by Struve (1944), Hill et al. (1974), Levato \& Morrell (1983), Levato et al. (1988), Perry et al. (1990), Penny et al. (1994), Stickland \& Lloyd (2001, IUE data), García \& Mermilliod (2001), Sana et al. (2002) and Sana (2005). Several authors also paid a special attention to particular objects, mainly binaries of which they performed a more detailed study. These objects are WR 79 (Lührs 1997), HD 152218 (Stickland et al. 1997), HD 152248 (Stickland et al. 1996; Penny et al. 1999; Sana et al. 2001; Sana et al. 2004), CPD -41 7742 (Sana et al. 2003, 2005a) and HD 152219 (Sana et al. 2006a).

Aside from these three main streams, several authors addressed specific aspects of the cluster that provide a useful complementary view. Among other topics, photometric variability of a few dozens of objects was investigated by Balona (1983), Balona \& Engelbrecht (1985), Balona (1992) and more recently by Arentoft et al. (2001). These studies allowed to detect several $\beta$ Cephei, a couple of $\delta$ Scuti and a few other variable stars, including a couple of eclipsing binaries. The binary fraction was estimated by Raboud (1996) and García \& Mermilliod (2001). Raboud derived a minimum binary frequency of $52 \%$ in his sample of 53 B-type stars with a spectral type between B1 and B9 while García \& Mermilliod (2001) obtained an extremely high frequency of $82 \%$ for stars earlier than $\mathrm{B} 1.5 \mathrm{~V}$ and, in particular, of $79 \%$ for the O-type stars of the cluster. Raboud \& Mermilliod (1998) showed evidence of mass segregation in NGC 6231, most probably related to the formation processes rather than to the dynamical evolution of the cluster. Proper motions were studied by Braes (1967) and Laval (1972) while most of the O-type stars of the clusters were included in the large ICCD Speckle campaign of Mason et al. (1998).

The distance modulus $(D M)$ of the cluster reported in the earlier literature ranges from 10.7 (Mermilliod 1981) to 11.9 (Houck 1956, 2300 pc - cited by Bok et al. 1966). In a more recent work, Perry et al. (1991) obtained $D M=11.50$ and 11.55 for Sco OB 1 and NGC 6231 respectively, with an uncertainty of about 0.32. Balona \& Laney (1995) derived $D M=11.08 \pm$ 0.05 for NGC 6231; Raboud et al. (1997), $11.2 \pm 0.4$; Sung et al. (1998), $11.0 \pm 0.07$ and Baume et al. (1999) $11.5 \pm 0.25$. The weighted mean of these five latter values gives $D M=11.07 \pm$ 0.04 , corresponding to an actual distance of $1637 \pm 30 \mathrm{pc}$. The same authors (but Sung et al.) respectively derived ages of $7.9 \pm 0.9 \mathrm{Myr}, 5 \pm 1 \mathrm{Myr}, 3.8 \pm 0.6 \mathrm{Myr}$ and 3 to $5 \mathrm{Myr}$. On the basis of the R-H $\alpha$ index, Sung et al. (1998) found 12 PMS stars plus 7 PMS candidates.

A controversial question is the probable differential reddening across the cluster. Such a differential reddening was first suggested by Breckinridge \& Kron (1963), outlining that the southern part of the cluster suffers a heavier reddening. Other authors (Shobbrook 1983; Perry et al. 1991; Balona \& Laney 1995) rather proposed a uniform reddening across the field. More recently, Raboud et al. (1997) and Sung et al. (1998) results strongly support the first idea of Breckinridge \& Kron (1963), and Sung et al. presented a map of the reddening distribution in NGC 6231. There seems to be an agreement in the early literature that most of the reddening occurs between a distance of 100 and 1300 pc. Based on FUSE observations, Marggraf et al. (2004) recently confirmed angular variations in the column density towards the core of the cluster. They reported that the absorption towards NGC 6231 occurs in several foreground clouds. The main absorption component lies in the Lupus cloud region at a distance of $150 \mathrm{pc}$, while the second one is probably in the vicinity of the Sco OB 1 shell surrounding NGC 6231. Finally, Crawford (2001) probed the structure of the interstellar $\mathrm{Na}$ I and K I towards the cluster and reached conclusions similar to those of Marggraf et al. (2004). Crawford also outlined that no clues of active shocks in the shell components could be found. Polarimetric observations were performed by Feinstein et al. (2003) who found evidence for a past supernova explosion in the cluster. These authors however suggested that their observations could also be explained by a bubble triggered by winds from hot stars.

Finally, we note that the Hipparcos parallaxes derived for NGC 6231 were known to be problematic with a negative mean value of $-0.8 \pm 0.4$ mas (Arenou \& Luri 1999). These results were recently revised by Makarov (2003a) who obtained $1.7 \pm$ 0.4 mas, corresponding to a distance modulus of $8.9 \pm 0.5$, however still far from the mean value obtained from the photometric studies.

Turning to the X-ray domain, NGC 6231 was observed by the ROentgen SATellite (ROSAT). Thirty-five objects were detected, mainly associated with the early-type stars of the cluster. Corcoran $(1996,1999)$ presented some results of this campaign as well as the X-ray light curve of three objects, namely HD 152218, HD 152248 and HD 152249. Only HD 152248 displayed clear variations of its flux. Finally a few objects were also observed at radio wavelength (Setia Gunawan et al. 2002, 2003) but only half of them were detected.

\section{Observations and data reduction}

\subsection{The XMM-Newton campaign}

The XMM-Newton campaign towards NGC 6231 has already been described in Sana et al. (2004). For the sake of completeness, we again give here a brief description of the X-ray observations. In September 2001, during satellite revolutions 319 to 321, the XMM-Newton observatory (Jansen et al. 2001) performed six successive exposures of an approximate duration of $30 \mathrm{ks}$. The field of view (FOV) was centered on the O7.5 III+O7 III colliding wind binary HD $152248\left(\alpha_{2000}=16^{\mathrm{h}} 54^{\mathrm{m}} 10^{\mathrm{s}} .06, \delta_{2000}=\right.$ $-41^{\circ} 49^{\prime} 30^{\prime} 1$; Sana et al. 2001), in the core of the cluster. Position angles (PAs) were very similar through the six exposures, ranging approximatively from 274.95 to 276.23 . All three EPIC instruments (Strüder et al. 2001; Turner et al. 2001) were operated in the Full Frame mode together with the Thick Filter to reject UV/optical light. The RGS spectrographs (den Herder et al. 2001) were run in the Standard Spectroscopic mode. Due to the brightness of the cluster objects in the FOV, the Optical Monitor (Mason et al. 2001) was switched off throughout the campaign. Table 1 provides the journal of the X-ray observations.

\subsection{Data reduction}

The EPIC Observation Data Files (ODFs) were processed using the XMM-Science Analysis System (SAS) v 5.4.1 implemented on our computers in Liège. We applied the emproc and epproc pipeline chains respectively to the MOS and pn raw data to generate proper event list files. No indication of pile-up was 
Table 1. Journal of the XMM-Newton observations of NGC 6231. Columns 2 and 3 give the spacecraft revolution number and the observation ID. The Julian Date (JD) at mid-exposure is reported in Col. 4. Columns 5 to 7 (resp. Cols. 8 to 10) list the performed (resp. effective - i.e. corrected for the high background periods) exposure times for the EPIC MOS1, EPIC MOS2 and EPIC pn instruments. The last column provides the position angle (PA) associated to the revolution. The total campaign duration is given in the last line of the table.

\begin{tabular}{|c|c|c|c|c|c|c|c|c|c|c|}
\hline \multirow[t]{2}{*}{ 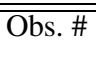 } & \multirow{2}{*}{$\begin{array}{l}\text { Sat. } \\
\text { Rev. }\end{array}$} & \multirow{2}{*}{$\begin{array}{l}\text { Exposure } \\
\text { ID }\end{array}$} & \multirow{2}{*}{$\begin{array}{c}\mathrm{JD} \\
\mathrm{JD}-2450000\end{array}$} & \multicolumn{3}{|c|}{$\overline{\text { Performed duration (ks) }}$} & \multicolumn{3}{|c|}{ Effective duration (ks) } & \multirow{2}{*}{$\begin{array}{c}\text { PA } \\
\text { DDD:AM:AS.s }\end{array}$} \\
\hline & & & & MOS1 & MOS2 & pn & MOS1 & MOS2 & $\mathrm{pn}$ & \\
\hline 1 & 319 & 0109490101 & 2158.214 & 33.3 & 33.3 & 30.7 & 33.1 & 33.2 & 30.6 & $274: 57: 11.5$ \\
\hline 2 & 319 & 0109490201 & 2158.931 & 22.1 & 22.1 & 20.2 & 19.8 & 19.8 & 16.5 & 274:57:11.5 \\
\hline 3 & 320 & 0109490301 & 2159.796 & 34.4 & 34.4 & 31.8 & 33.7 & 33.9 & 30.1 & $275: 35: 26.6$ \\
\hline 4 & 320 & 0109490401 & 2160.925 & 31.4 & 31.4 & 29.1 & 26.0 & 24.3 & 11.7 & $275: 35: 26.6$ \\
\hline 5 & 321 & 0109490501 & 2161.774 & 31.1 & 31.1 & 28.5 & 30.9 & 31.0 & 28.4 & $276: 13: 34.9$ \\
\hline 6 & 321 & 0109490601 & 2162.726 & 32.9 & 32.9 & 30.3 & 32.9 & 32.8 & 30.3 & $276: 13: 34.9$ \\
\hline \multicolumn{4}{|c|}{ Total duration (ks) } & 185.2 & 185.2 & 170.6 & 176.5 & 175.0 & 147.5 & \\
\hline
\end{tabular}

found in the data. We then only considered events with patterns 0-12 (resp. 0-4) for MOS (resp. pn) instruments and we applied the filtering criterion XMMEA_EM (resp. FLAG =0) as recommended by the Science Operation Centre (SOC) technical note XMM-PS-TN-43 v3.0. For each pointing, we rejected periods affected by soft proton flares. For this purpose, we built light curves at energies above $10 \mathrm{keV}^{1}$ and discarded high background observing periods on the basis of an empirically derived threshold (adopted as equal to 0.2 and $1.0 \mathrm{cnt} \mathrm{s}^{-1}$ for the MOS and pn instruments respectively). The so-defined GTIs (Good Time Intervals) were used to produce adequate $\mathrm{X}$-ray event lists for each pointing from which we extracted images using $x$ - and $y$-image bin sizes of 50 virtual pixels ${ }^{2}$.

We finally combined the event lists obtained for all six pointings to increase the statistics of faint sources. For this purpose, we used the SAS task merge. For each EPIC instrument, we included the event lists resulting from different pointings one by one. We also built merged event lists that combine the twelve MOS or the eighteen EPIC event lists. The Attitude Files generated by the pipeline were merged using the same approach and we adopted, for handling the merged event lists, the Calibration Index File (CIF) and the ODF corresponding to the first pointing (Obs. 1 in Table 1).

\section{X-ray source detection and identification}

In this section, we focus on the detection and identification of the X-ray sources in the XMM-Newton FOV. For this purpose, we only used the merged event lists and images, accounting in this way for the six pointings at once. The total effective exposure times towards the cluster are, respectively for the MOS1, MOS2 and pn instruments, of $176.5,175.0$ and $147.5 \mathrm{ks}$. Together with the high sensitivity of the XMM-Newton observatory, the combination of the six pointings and of the three instruments provides one of the deepest X-ray views of a young open cluster. Figure 1 shows a three-colour image of NGC 6231 and reveals a densely populated field with hundreds of point-like X-ray sources. This section therefore aims at providing a uniform catalogue of these sources. It is organised as follows. First we present the source detection procedure as well as a brief description of the obtained catalogues. As a next step, we focus on the identification of the

\footnotetext{
${ }^{1}$ Expressed in Pulse Invariant (PI) channel numbers and considering that $1 \mathrm{PI}$ channel approximately corresponds to $1 \mathrm{eV}$, the adopted criterion is actually PI $>10000$.

2 Though the physical pixels of the EPIC MOS and pn detectors have an extent on the sky of respectively $11^{\prime \prime} 1$ and $4{ }^{\prime \prime} 1$, the virtual pixels of the three instruments correspond to an extent $0{ }^{\prime} 05$. The obtained images have thus a pixel size of 2 ". 5 .
}

X-ray sources and, finally, we investigate the detection limit of the present data set.

\subsection{Source detection}

We based our source detection on the SAS detection chain edetect_chain. For this purpose, we selected three energy ranges, a soft $\left(S_{\mathrm{X}}\right)$ band $(0.5-1.0 \mathrm{keV})$, a medium $\left(M_{\mathrm{X}}\right)$ band $(1.0-2.5 \mathrm{keV})$ and a hard $\left(H_{\mathrm{X}}\right)$ band $(2.5-10.0 \mathrm{keV})$, and we built the corresponding input images for the different instruments. The edetect_chain task is formed by the succession of the SAS tasks eexpmap, emask, eboxdetect run in local mode, esplinemap, again eboxdetect run in map mode and finally emldetect:

- eexpmap calculates the exposure maps corresponding to the input images;

- these exposure maps are used by emask to build masks which select the relevant image areas where the detection should take place;

- eboxdetect, run in local mode, uses a $5 \times 5$ pixel box and a surrounding background area to search for significant sources simultaneously in all input images;

- esplinemap uses the resulting source list to remove sources from the input images and creates smooth background maps by fitting a $2 \mathrm{D}$ spline to the source-subtracted images;

- run in map mode, eboxdetect uses a $5 \times 5$ pixel box and the values from the background maps to search for significant sources simultaneously in all input images;

- emldetect finally uses the preliminary source list from eboxdetect and determines the source parameters (e.g. coordinates, count rates, hardness ratios, etc.) by means of simultaneous maximum likelihood psf (point spread function) fitting to the source count distribution in all energy bands of each EPIC instrument. It also provides an equivalent logarithmic likelihood $L_{2}$ (Eq. (A.1)) commonly used as an indication of the reality of the corresponding source.

From our experience, the eboxdetect task run in map mode tended to eliminate some apparently real sources from the intermediate source list. We therefore preferred to use the preliminary source list obtained by eboxdetect in local mode as an input list for the psf fitting step performed by the emldetect task. This approach does not bias the result since, if the source is real, the psf fitting will provide a large logarithmic likelihood while, if instead the source is fake, the logarithmic likelihood will be low and the source will be rejected. Though more expensive in computation time, this approach results in a more complete source list. As it was known that the equivalent logarithmic likelihood values $\left(L_{2}\right)$ computed by the emldetect task in the SAS v 5.4.1 


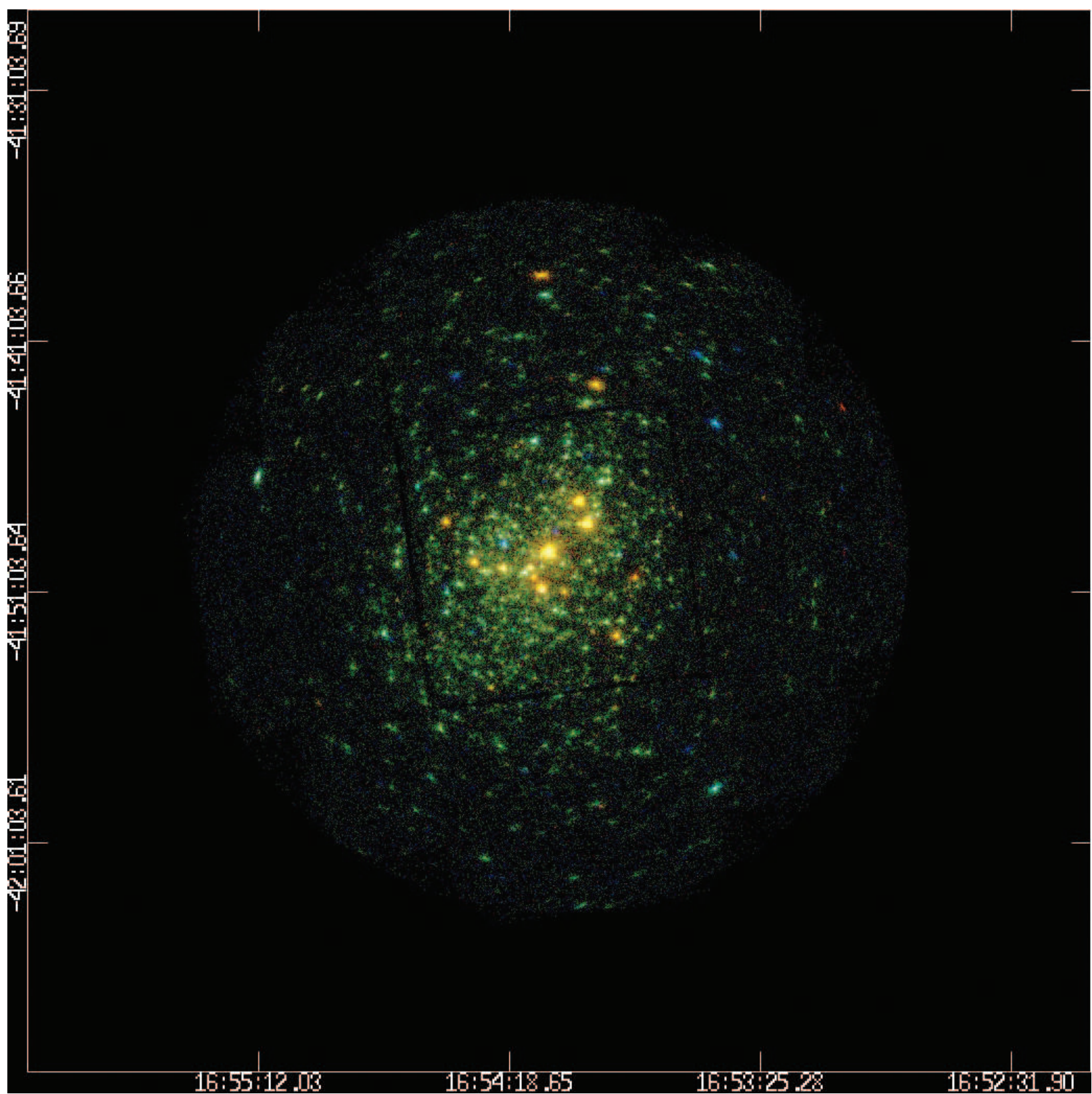

Fig. 1. Combined EPIC MOS three-color X-ray image of the young open cluster NGC 6231. The field is roughly $30^{\prime}$ in diameter and is centered on HD 152248. North is up and East is to the left. The different colors correspond to different energy ranges: red: $0.5-1.0 \mathrm{keV}$; green: 1.0-2.5 keV; blue: $2.5-10.0 \mathrm{keV}$.

(and earlier versions) were erroneous, we implemented a patch to recover the correct $L_{2}$ values. We give a brief description of it in Appendix A. The problem is now fixed from SAS version 6.0 on. We checked our corrected logarithmic likelihood values against SAS v 6.0 results and found them to be in close agreement.

We first performed single psf fit detection but, due to the crowdedness of the field, we also allowed for simultaneous fitting of up to four sources. In doing so, we adopted a value of 0.68 for the two parameters scut and ecut. This choice was led by the need to account for as large an energy fraction of the psf as possible while keeping the computation time down to reasonable limits. Due to the densely populated field, the wings of the source psf are often largely contaminated by emission from neighbouring sources. The adopted values therefore appeared as a reasonable compromise. On the axis, this corresponds to a physical radius of about $15^{\prime \prime}$. Only a few tens of sources actually required multi-psf fitting, with three psf being simultaneously adjusted at the maximum. Finally, we re-ran the emldetect task allowing for extended sources to be fitted. A careful comparison of the resulting lists shows that only a few sources increase significantly their detection likelihood while allowing for extended source fitting. An inspection of the X-ray images and of the optical catalogues reveals that these sources most probably correspond to unresolved point-like sources rather than to physically extended sources.

The described detection procedure was applied for each EPIC instrument as well as for any combination of them. The resulting source lists were generally consistent. The main difference comes from the presence of different gaps in the different data sets. We built our final source list adopting the following criteria. 
Table 2. Adopted detection thresholds for the equivalent logarithmic likelihood $L_{2}$ corresponding to the different EPIC instruments (left column) or to any combination of them (right column). Appendix B provides more details on how these values were computed.

\begin{tabular}{cc|cc}
\hline \hline Instr. & $L_{2}$ & Instr. comb. & $L_{2}$ \\
\hline MOS1 & 11 & MOS1 + MOS2 & 21 \\
MOS2 & 11 & MOS1 + pn & 35 \\
pn & 25 & MOS2 + pn & 35 \\
& & MOS1 + MOS2 + pn & 45 \\
\hline
\end{tabular}

(i) We selected the deepest combination of EPIC instruments, requiring that the detected source is distant by at least $13^{\prime \prime}$ from any gap, bad column or detector edge.

(ii) By a visual inspection of each source in all images and subsequent combinations, we adopted an empirical equivalent logarithmic likelihood $\left(L_{2}\right)$ threshold as the detection limit. This led us to consider the way to perform a consistent choice for the threshold values while dealing with different instrument combinations, and hence with different numbers of input images. As a general comment, it is obvious that adopting a constant logarithmic likelihood threshold while dealing with different combinations of the EPIC instruments does not allow us to keep a constant threshold in terms of the signal level. Indeed, all other things being equal, the signal-to-noise ratio is increased while combining several detectors, allowing us in principle to detect fainter sources. However, in such a crowded field as the current one, we note that no important gain is achieved in terms of source detection. In other words, the very large majority of the detected sources are already seen in a single instrument, though of course combining the different instruments yields a much better estimation of their X-ray parameters. As a consequence, we have decided to adopt a logarithmic likelihood threshold in one instrument and to look for the equivalent thresholds in any EPIC combination. This issue is presented into more details in Appendix B. Table 2 gives the logarithmic likelihood thresholds finally used for the source detection. These values provide thresholds in various combinations that are consistent with the logarithmic likelihood-based detection threshold adopted in a single MOS instrument. We note that this procedure does not modify the spatial response of the detectors and that the known variations of the EPIC instrument sensitivity with the axial distance will of course still affect our results.

(iii) In the few cases for which multi-source fitting was relevant, we adopted the results obtained with this fitting. We however paid a special attention to reject cases of fake multifitting sometimes induced by near-gap/edge effects or by multiple entries for a unique X-ray source in the preliminary source list.

(iv) We finally checked every source in the final list by individually looking at the different image combinations. We eliminated the very few double entries in the list. Doing this, we noticed a couple of presumably physical sources that were ignored by the detection algorithm. We decided to include those sources in the input source list of the emldetect task. Most of them were satisfactorily fitted, giving an equivalent logarithmic likelihood above the adopted detection threshold. These additional sources were included in the final catalogue.

(v) The main X-ray catalogue presented in Table 3 is based on the point-like source detection only. For some sources, the equivalent logarithmic likelihood $L_{2}$ is significantly increased if one adjusts an extended source model rather than a point-like model. These sources are flagged in Table 3 and we provide, in Table 4, a complementary extended source catalogue that gives, in addition to the results listed in the main catalogue, the emldetect extended-psf fit results for these sources.

The final catalogue lists 610 sources in the XMM-Newton FOV, among which 19 are flagged as extended. Based on the edetect_chain results, it provides, among other information, the source position, the total count rates in the different instruments and the two hardness ratios:

$$
\begin{aligned}
& H R_{1}=\frac{M_{\mathrm{X}}-S_{\mathrm{X}}}{M_{\mathrm{X}}+S_{\mathrm{X}}}, \\
& H R_{2}=\frac{H_{\mathrm{X}}-M_{\mathrm{X}}}{H_{\mathrm{X}}+M_{\mathrm{X}}} .
\end{aligned}
$$

A sample of the catalogue is provided in Table 3 while Table 4 gives the complementary catalogue for the 19 extended X-ray sources detected. In addition, source X\#234 appears clearly double in the EPIC image though it is not detected as an extended object. Table 3 is available online via the Centre de Données astronomiques de Strasbourg. Finding charts for the $\mathrm{X}$-ray sources are provided by Figs. 2 and 3.

\subsection{Source identification}

To determine the optical counterparts of the detected X-ray sources, we cross-correlated our source list with several existing optical/infrared catalogues. We used the US Naval Observatory (Monet et al. 2003, USNO B1.0), the 2MASS All Sky Data Release (Cutri et al. 2003) and the Guide Star Catalogue-II (GSC 2.2 2001). We also made use of the optical catalogue of Sung et al. (1998, SBL98 hereafter). However, the star positions in the SBL98 catalogue as available from the Centre de Données astronomiques de Strasbourg (CDS) show clear shifts compared to the true positions on the sky. This results from an excessive rounding of the star coordinates in the CDS database: they are given with a precision of respectively one second and one tenth of arcmin on the right ascension and declination. This is far insufficient in such a crowded field as NGC 6231. We therefore used the original SBL98 catalogue, obtained from the authors and that lists object coordinates a hundred times more precisely. Beyond the 860 objects with $V \leq 16$ listed in SBL98, this $U B V(R I)_{\mathrm{C}} \& \mathrm{H} \alpha$ catalogue was completed with 7199 objects, extending the first version of the SBL98 catalogue down to $V=21$. However, the SBL98 field of view was limited to a $20^{\prime} \times$ $20^{\prime}$ area and thus does not cover the whole EPIC FOV. It can thus not be used for identification throughout the entire field and we selected the X-ray sources that are located in the sub-region of the FOV that is covered by the SBL98 v2 catalogue. This yields a number of X-ray sources $N_{\mathrm{X}}^{\mathrm{SBL}}=536$ as quoted in Table 5 . More recently, one of us (H. Sung) acquired new $U B V(R I)_{\mathrm{C}}$ observations covering a field of about $40^{\prime} \times 40^{\prime}$ around NGC 6231 . 30866 stars were observed down to $V<22$. These observations will be presented in a forthcoming paper (Sung et al. 2006, in preparation) and we only focused here on the resulting catalogue. We will refer to this new catalogue as the SSB06.

As a first approach, we investigated the possibility of systematic differences between the reference frames of the different catalogues. For this purpose, we selected the bright O-type stars in the different source lists and we compared their locations to the ones of their X-ray counterparts. Neither a significant systematic 


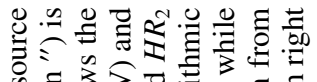

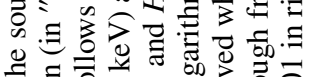

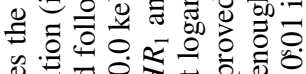

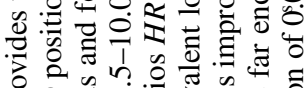
है

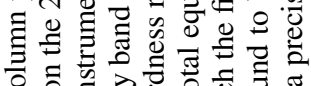

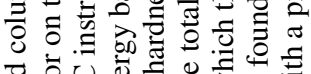

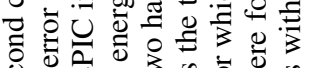

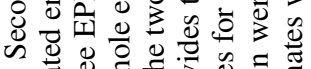

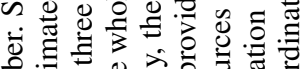

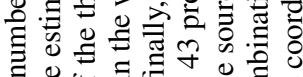

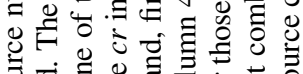

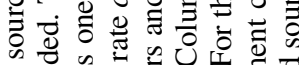

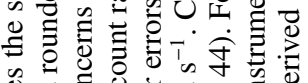
$\bigodot_{00}^{0} 0$ 。

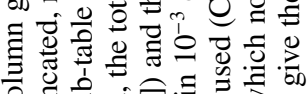

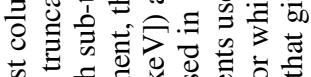

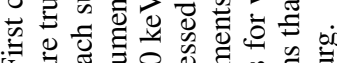

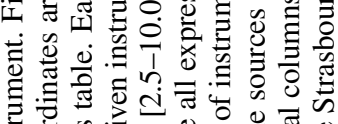

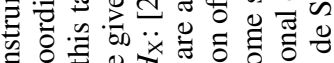

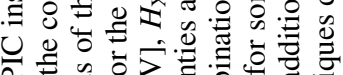

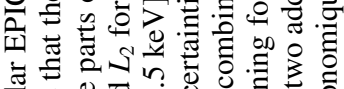

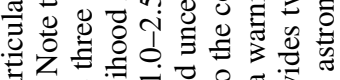

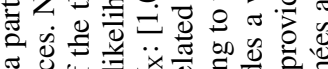

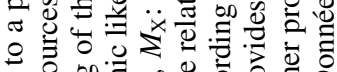
no

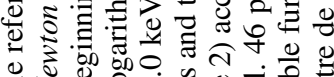

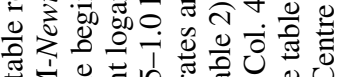

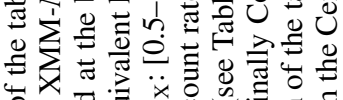

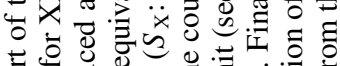

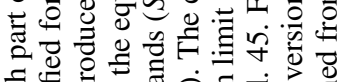

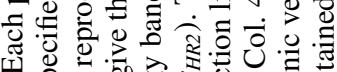
D क्र 0 on

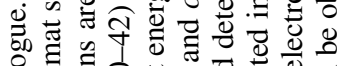

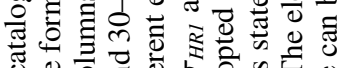

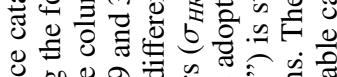

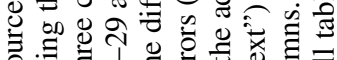

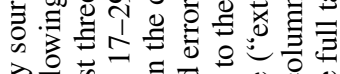

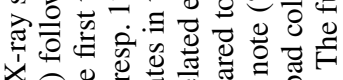

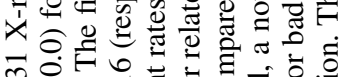

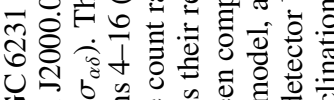

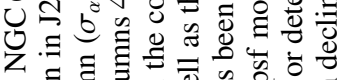

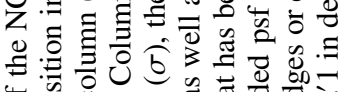

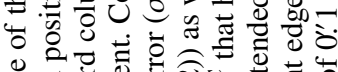

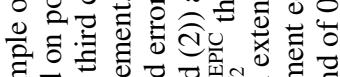

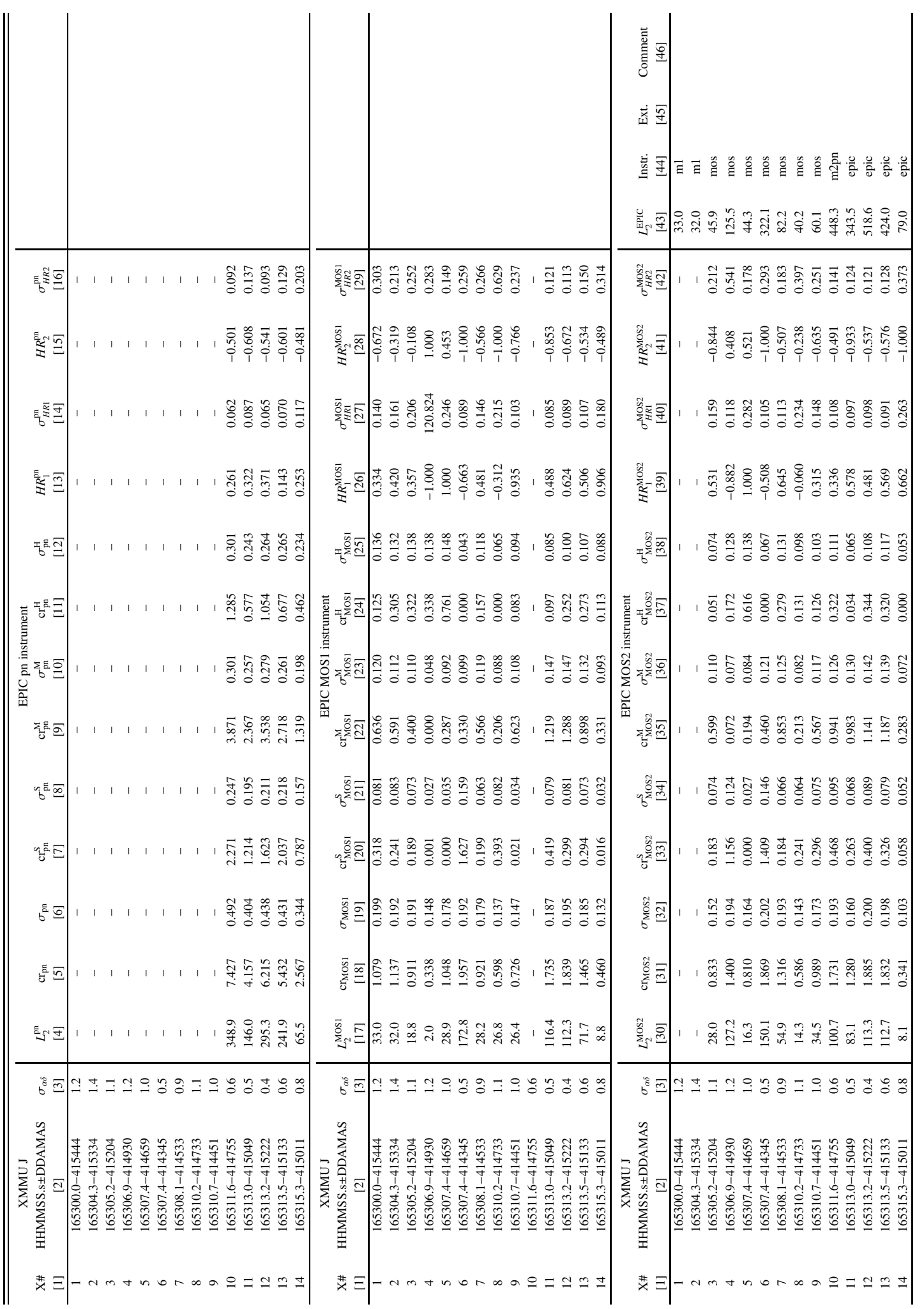

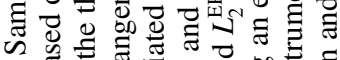

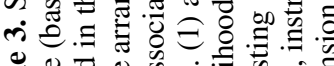

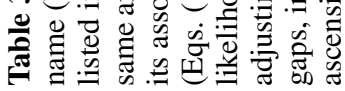




\begin{tabular}{|c|c|c|c|c|c|}
\hline & & & & 音表 & 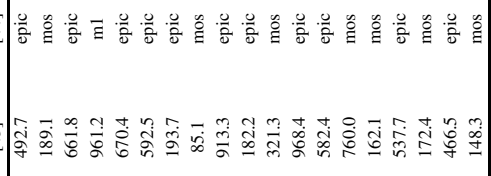 \\
\hline 톤은 & 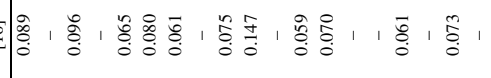 & 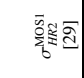 & 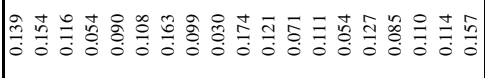 & & 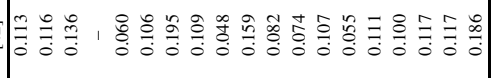 \\
\hline 高包 & 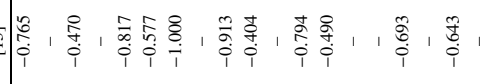 & & 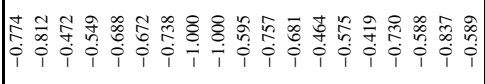 & & 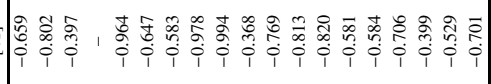 \\
\hline 㩊王 & 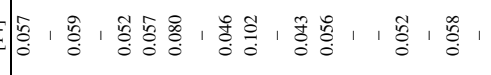 & 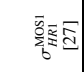 & 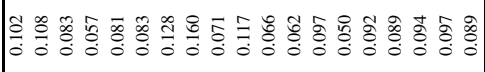 & & 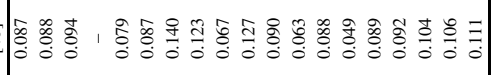 \\
\hline$\stackrel{\overline{3}}{3}$ & {$\left[\begin{array}{ll}\infty \\
\infty \\
0\end{array}\right.$} & 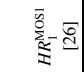 & 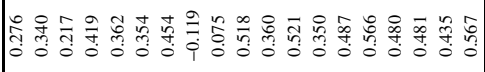 & & 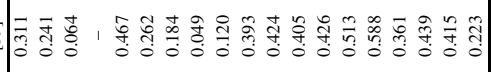 \\
\hline $\mathrm{r} \overline{\mathrm{g}} \overline{\mathrm{g}} \mathrm{g}$ & 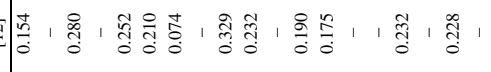 & 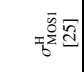 & 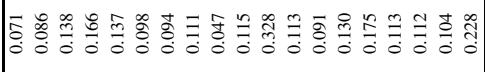 & & 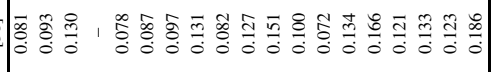 \\
\hline 좋 & 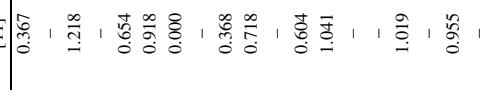 & 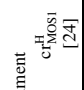 & 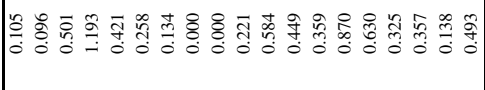 & 旋 & |⿹丁口八 \\
\hline 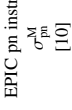 & 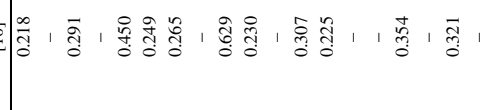 & 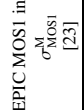 & 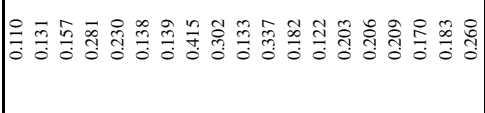 & 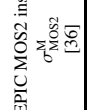 & 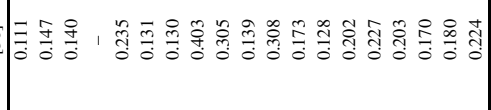 \\
\hline 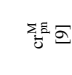 & 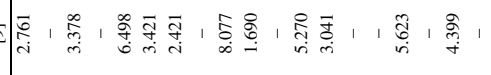 & 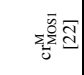 & 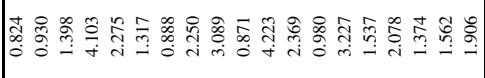 & & 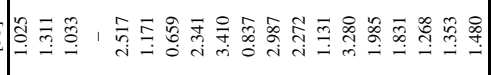 \\
\hline$b^{\bar{a}} \underline{\alpha}$ & 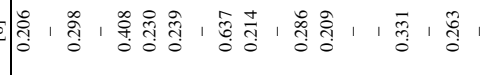 & $\sin _{0}^{\overline{\frac{s}{2}}} \overline{\bar{d}}$ & 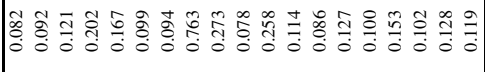 & & 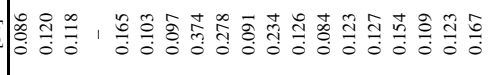 \\
\hline 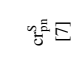 & 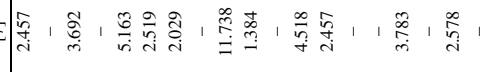 & 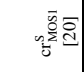 & 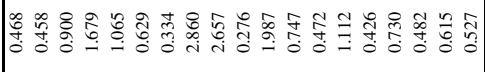 & & 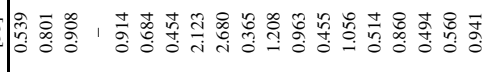 \\
\hline$\underline{0}$ & 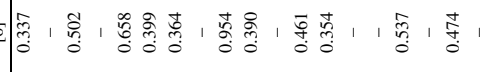 & $\frac{\bar{s}}{2} \bar{\Xi}$ & 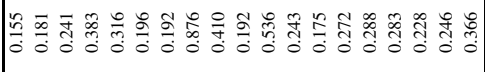 & & 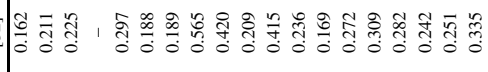 \\
\hline हू & 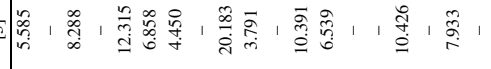 & $\stackrel{\bar{s}}{\overline{2}}=$ & 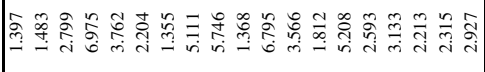 & & 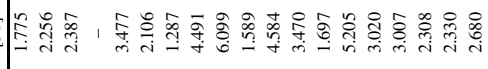 \\
\hline 脣す & 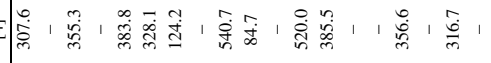 & 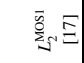 & 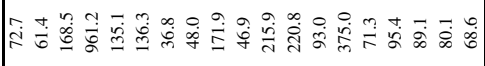 & 迩司 & 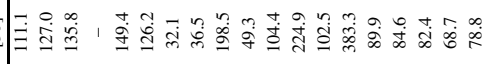 \\
\hline 产递 & 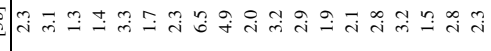 & 吾通 & 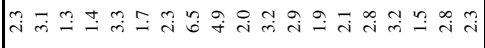 & 害远 & 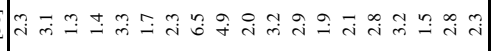 \\
\hline 疍器 & {$\left[\begin{array}{lll}\infty \\
\hdashline\end{array}\right.$} & $b^{8}$ 㩊 & 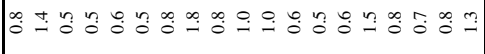 & 昜 哥 & 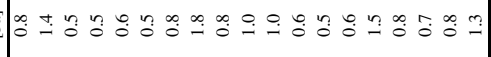 \\
\hline 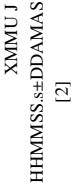 & 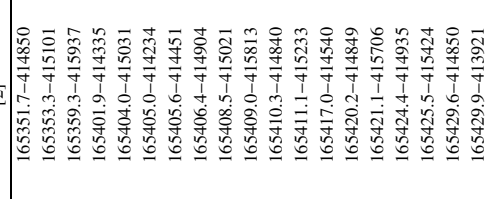 & 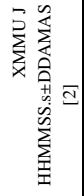 & 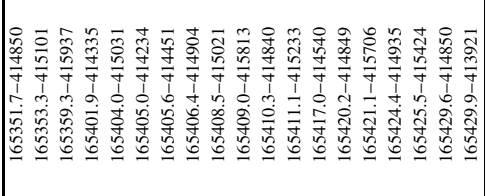 & 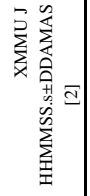 & 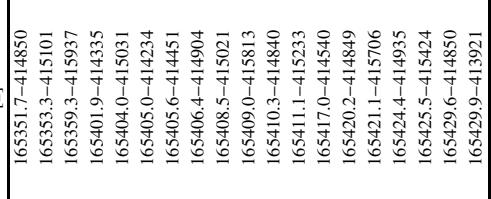 \\
\hline 芝 $\Xi$ & 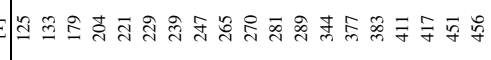 & 芝 $\Xi$ & ఇ̊心 & 芝 $\Xi$ & 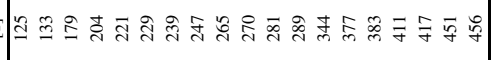 \\
\hline
\end{tabular}




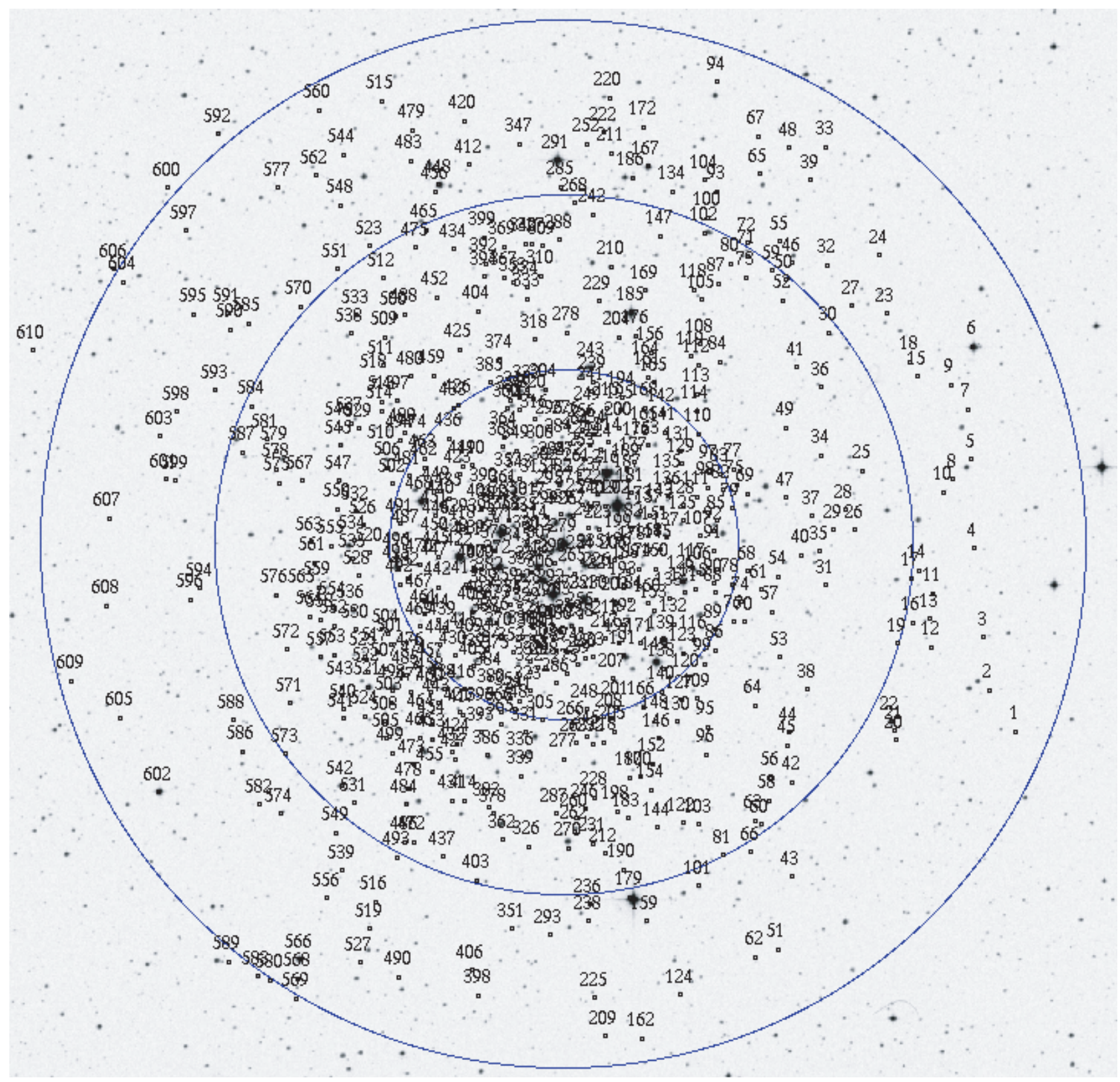

Fig. 2. Detected X-ray sources overlaid on a DSS1 image ( $V$ band) of the XMM-Newton FOV. The sources are indicated by black circles, with a radius of $3^{\prime \prime}$, similar to the adopted cross-correlation radius. The numbers above these circles give the internal X-ray source identification as provided in the first column of Table 3 . The three blue circles indicate regions with a radius of $5^{\prime}, 10^{\prime}$ and $15^{\prime}$ around X\# 279 (HD 152248 ). North is up and East is to the left.

shift nor a field rotation was apparent. Typical 1- $\sigma$ dispersions computed on the differences between the locations of the X-ray sources and their optical counterparts are about $0.9^{\prime \prime}$ in right ascension and $0.7^{\prime \prime}$ in declination. Similarly, the $1-\sigma$ dispersion on the field rotation is about $3^{\prime}$. As a second step and for each of the previously cited catalogues, we determined the closest optical counterpart of each X-ray source in the field of view. We then calculated the cumulative distribution $(\Phi(r))$ of the closest associated counterparts as a function of the individual correlation radius (see Jeffries et al. 1997). The generated diagrams are shown in Fig. 4. Following Jeffries et al., we assumed that $\Phi(r)$ is formed by two terms: the cumulative distribution of true correlations $\Phi_{\text {true }}$ and the cumulative number of spurious associations $\Phi_{\text {spur }}$. This is expressed in the simple relation:

$\Phi(r)=\Phi_{\text {true }}+\Phi_{\text {spur }}$

$$
=A\left[1-\exp \left(\frac{-r^{2}}{2 \sigma^{2}}\right)\right]+\left(N_{\mathrm{X}}-A\right)\left[1-\exp \left(-\pi r^{2} B\right)\right]
$$

that can be adjusted to the empirical distribution. In Eq. (4), $N_{\mathrm{X}}$ is the number of X-ray sources while $A$ is the number of true correlations with the optical/infrared catalogue. $B$ is the optical/infrared catalogue density and $\sigma$ is related to the statistical uncertainty on the X-ray source position. Though Eq. (4) is approximative and rests on the hypothesis of a uniform optical population (i.e. constant $B$ and constant psf over the full FOV), it fits reasonably well the rising branch of the different curves plotted in Fig. 4. Table 5 gives the obtained values of the $A, B$ and $\sigma$ parameters. As the hypothesis of constant $B$ throughout the FOV is clearly violated in the case of NGC 6231, we also estimated the number of spurious associations using a more empirical approach. We arbitrarily shifted the X-ray source positions 


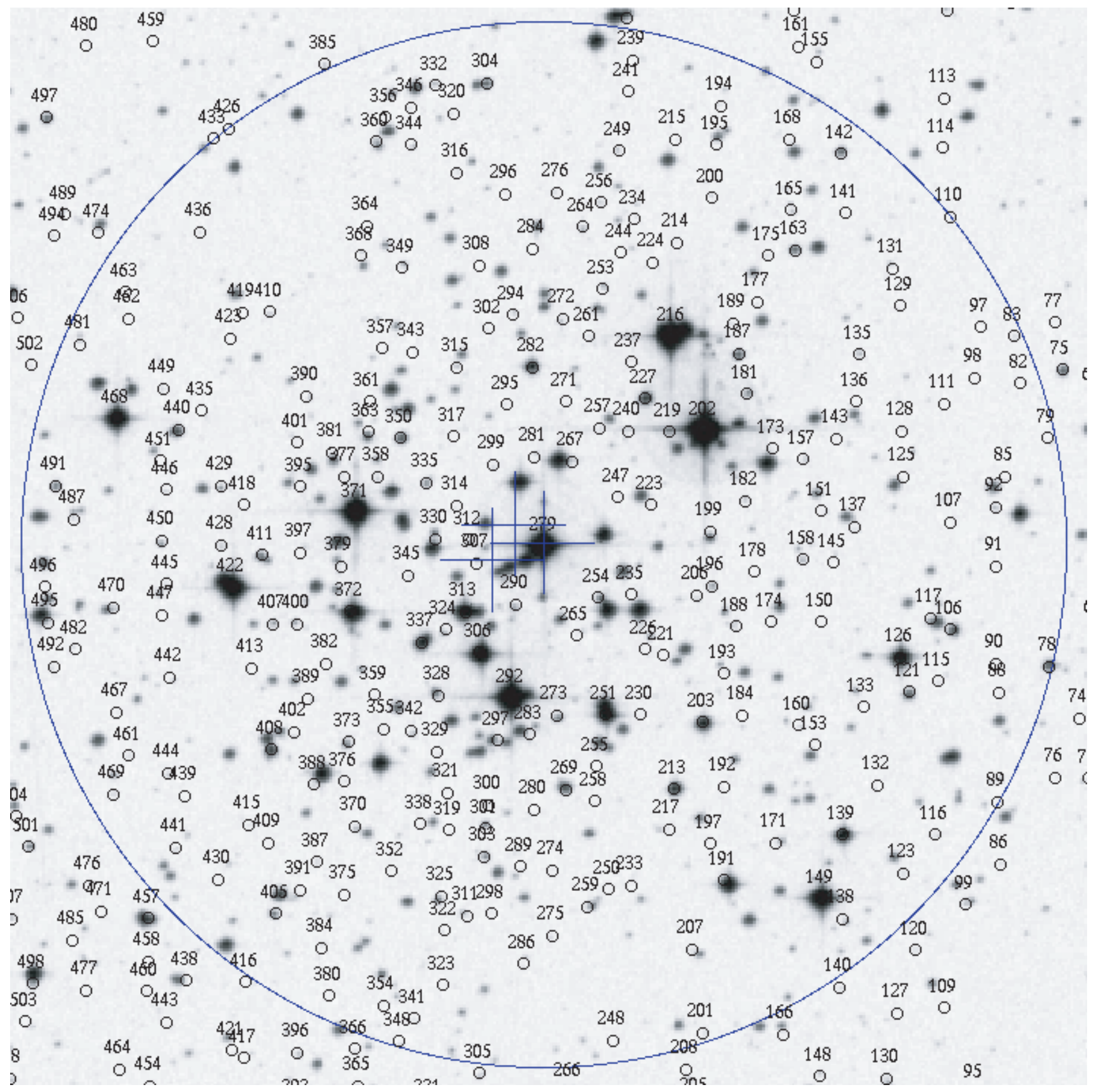

Fig. 3. Same as Fig. 2, zoomed on the inner part of the FOV. The circle radius is $5^{\prime}$. From left to right, the three crosses respectively give the position of the geometrical centre of the cluster, its X-ray emission centre (computed adopting the pn-equivalent count rates for each source), and the position of HD 152248 (X\# 279).

by $30^{\prime \prime}$ in any given direction and we re-ran the cross-correlation at a fixed $r_{\text {corr }}$ (either $2^{\prime \prime} 5$ or $3{ }^{\prime \prime} .0$ according to the catalogue considered). The obtained number of spurious associations is never larger by more than $10 \%$ than the one estimated by the Jeffries et al. (1997) method.

The SSB06 catalogue is too dense for the relative crudeness of the X-ray source positions $\left(\sigma_{\alpha \delta}=0.7 \pm 0 . ' 3\right.$ on average, $\sigma_{\alpha \delta}$ being defined on 2D). Indeed, even adopting a limited cross-correlation radius of 2'.5 would yield over 100 spurious identifications. We thus decided to decrease the limiting magnitude of the catalogue. The maximum of the $\phi_{\text {true }}$ function is obtained adopting $V<20$. At the distance of the cluster, this corresponds to the magnitude of a M0 dwarf $\left(M \sim 0.5 M_{\odot}\right)$. PMS low-mass stars being brighter than ZAMS stars of the same mass, the progenitors of M0 stars should thus still be included in the optical list. Finally, we note that a significant improvement (in terms of the relative percentage of spurious associations) is obtained when restricting the SSB06 cross-correlation to objects with $V<19$. The drawback is that the number of true associations is also significantly reduced. Table 5 lists the best fit parameters of Eq. (4) for both cases and Table 3 provides the SSB06 cross-identifications down to $V<20$. We leave to the user the choice to restrict the list to $V<19$ according to his/her motivations.

From the cumulative distributions shown in Fig. 4, we adopted the cross-correlation radii corresponding to the knees in the distributions of counterparts; these are reported in Table 5. The percentage of identified sources ranges from 55 to $83 \%$ 
Table 5. Best fit parameters (Cols. 3-5) of the $\Phi(r)$ function (Eq. (4)) for different optical/infrared catalogues. The catalogue name is given in Col. 1 along with the relevant number of X-ray sources in the corresponding field (Col. 2). The adopted correlation radius $\left(r_{\text {corr }}\right)$ for identification is given in Col. 6. Column 7 lists the actual number $N_{\text {corr }}$ of identified X-ray sources (see Table 6) and the corresponding percentage related to the considered number of X-ray sources $\left(N_{\mathrm{X}}\right)$. Column 8 gives the number of associated counterparts predicted by the distribution $\Phi(r)(\mathrm{Eq}$. (4)) at a radius equal to $r_{\text {corr }}$. It also provides the corresponding percentage of theoretically identified X-ray sources. The next two columns provide, among the number of associated counterparts $\Phi\left(r_{\text {corr }}\right)$, the predicted number of true $\left(\Phi_{\text {true }}\left(r_{\text {corr }}\right)\right)$ and spurious $\left(\Phi_{\text {spur }}\left(r_{\text {corr }}\right)\right)$ counterparts. The contribution of true and spurious counterparts to the total number of (theoretically) associated optical sources are also given in the corresponding columns.

\begin{tabular}{cccccccccc}
\hline \hline Opt. cat. & $N_{\mathrm{X}}$ & $A$ & $\begin{array}{c}\sigma \\
\left({ }^{\prime \prime}\right)\end{array}$ & $\begin{array}{c}B \\
10^{-3}\left({ }^{\prime \prime}\right)^{-2}\end{array}$ & $\begin{array}{c}r_{\text {corr }} \\
\left({ }^{\prime \prime}\right)\end{array}$ & $N_{\text {corr }}$ & $\Phi\left(r_{\text {corr }}\right)$ & $\Phi_{\text {true }}\left(r_{\text {corr }}\right)$ & $\Phi_{\text {spur }}\left(r_{\text {corr }}\right)$ \\
\hline 2MASS & 610 & 322.2 & 0.91 & 30.0 & 2 & $384(63.0 \%)$ & $383.8(62.9 \%)$ & $293.3(76.4 \%)$ & $90.5(23.6 \%)$ \\
GSC 2.2 & 610 & 384.3 & 1.25 & 3.5 & 3 & $372(61.0 \%)$ & $383.7(62.9 \%)$ & $362.7(94.5 \%)$ & $21.1(5.5 \%)$ \\
USNO & 610 & 383.1 & 1.24 & 0.8 & 3 & $344(56.4 \%)$ & $367.8(60.3 \%)$ & $362.8(98.6 \%)$ & $5.0(1.4 \%)$ \\
SBL98 v2 & 536 & 431.8 & 1.09 & 11.9 & 3 & $447(83.4 \%)$ & $451.9(84.3 \%)$ & $422.1(93.4 \%)$ & $29.8(6.6 \%)$ \\
SSB06: $V<19$ & 610 & 396.6 & 1.01 & 4.3 & 2.5 & $384(63.0 \%)$ & $395.7(64.9 \%)$ & $378.4(95.6 \%)$ & $17.3(4.4 \%)$ \\
SSB06: $V<20$ & 610 & 422.6 & 0.95 & 13.9 & 2.5 & $450(73.8 \%)$ & $453.7(74.4 \%)$ & $408.9(90.1 \%)$ & $44.9(9.9 \%)$ \\
\hline
\end{tabular}
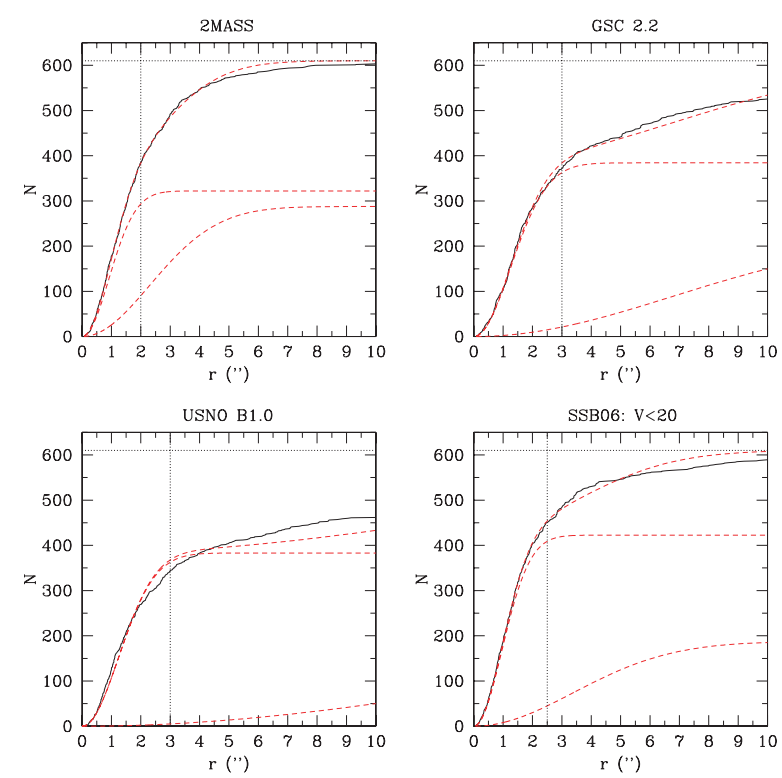

Fig. 4. Cumulative distributions (solid lines) of the number $(N)$ of closest associated counterparts as a function of the separation radius $(r)$ and for the different catalogues used. The horizontal dotted lines show the number $N_{\mathrm{X}}$ of X-ray sources in the catalogue. The dashed lines, from top to bottom in each panel, correspond respectively to the best-fit $\Phi(r)$ function, to the number of truly associated counterparts $\Phi_{\text {true }}$ and to the number of spurious ones $\Phi_{\text {spur }}$, as a function of the correlation radius $r$. Dotted vertical lines show the correlation radius adopted for the purpose of source identification.

according to the catalogue used. The results for the SBL98 v2 and the SSB06 catalogues are clearly in contrast with the other catalogues. With about $75 \%$ of the total number of X-ray sources in the FOV being identified, among which less than $10 \%$ statistically correspond to spurious associations, the latter catalogue is probably the most appropriate for the identification processes. In the following, we thus adopt the SSB06 catalogue, that covers the complete EPIC FOV, as the main reference in the identification of the sources. Table 6 provides the cross identifications between the X-ray source lists and the different optical catalogues. We find at least one counterpart in one catalogue for about $85 \%$ of the X-ray sources.

While carrying out this work, we noticed some confusion between the names of several sources reported in the widely consulted SIMBAD database. For this reason, Table 6 also gives other commonly adopted source denominations such as HD, CPD and Braes numbers. The Seggewiss numbering is also extensively used in the literature related to NGC 6231. We therefore used the original chart of Seggewiss (1968b) - subsequently completed by Raboud et al. (1997) - and we rederived the crosscorrelation to avoid any previous misidentification.

\subsection{The detection limit}

This paragraph aims at the evaluation of the detection limit of the present X-ray catalogue. Though essential, this question is far from trivial because the detection limit is, a priori, not uniform throughout the field of view. Besides the areas where the detectors do not overlap and the presence of gaps between the detector CCDs, the XMM-Newton effective exposure duration is decreasing from the FOV centre towards its edges. In addition, both the crowdedness of the field in its central part and the numerous bright sources preferentially located in the core of the cluster also affect the detection limit in a non uniform way. As an approximation we neglect the effects of the gaps, mainly focusing on the three other effects.

The exposure maps computed for the three EPIC instruments and their different combinations display a smooth decrease from the centre of the detector to its edges by about a factor of three. In terms of the amount of signal collected for two similar sources one located near the FOV axis, the other near one of its edges the number of counts $n$ received will be three times higher near the axis. Neglecting any background effect, the signal-to-noise ratio is approximately given by $S / N=\sqrt{n}$. For the outer source, it is therefore smaller by a factor of $\sqrt{3} \approx 1.7$. To the first order, the detection limit in the outer parts of the field is thus about a factor two higher than in the central part of the FOV. As a next step, we used the SAS task esensmap to build sensitivity maps corresponding to the current exposure maps and to the adopted logarithmic likelihood detection thresholds $L_{2}$. The sensitivity maps obtained actually provide the minimum number of counts for a source to be detected by the detection task emldetect according to the given equivalent logarithmic likelihood threshold. These maps indeed predict that the sensitivity of the EPIC camera is twice larger near the axis than in the outer parts of the detector whatever the instrument combination is. This is in agreement with our previous estimate.

Accounting for the variation of the source density and the distribution of the bright sources in the FOV is a more tricky issue. We chose to adopt a completely empirical approach, taking advantage of the large number of X-ray sources in the field. We assumed that a very good indication of the detection limit in the different parts of the field is given by the brightness of the faintest sources detected in these selected areas. 


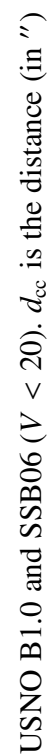

ปี

Uू.

䆓:

$\sum_{i}$

$\ddot{\leftrightarrow}$

등

密

蛋.

记

들

당

훙ํㅇ

要焉

흠

政

象

漹

을

品

焉然

:

产 홍

矛兽

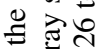

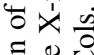

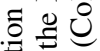

可

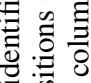

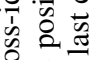

80

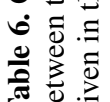

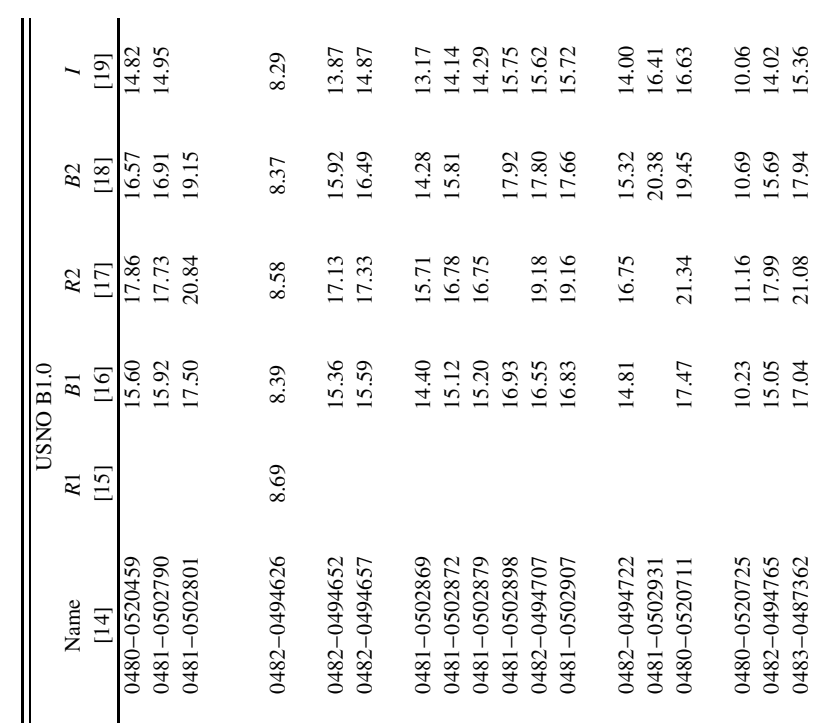

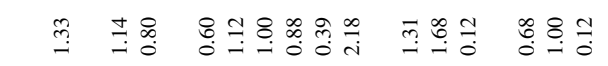

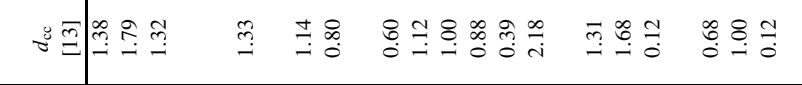

$>$ a

$\underset{\infty}{\infty}$

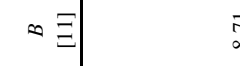

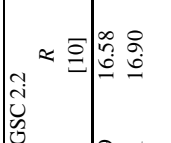

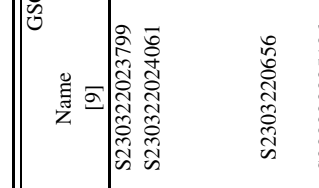

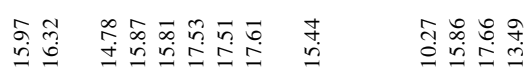

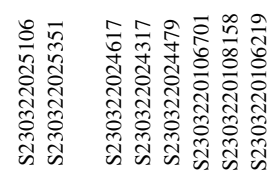

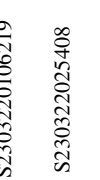

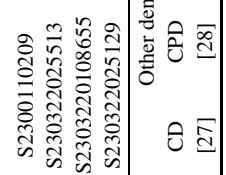

\begin{tabular}{|c|c|c|c|}
\hline 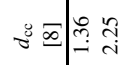 & $\stackrel{m}{g}:$ & 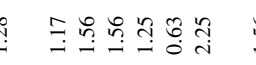 & 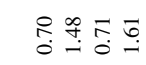 \\
\hline
\end{tabular}

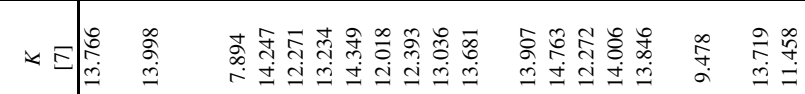

$=5$

$=0$ 造

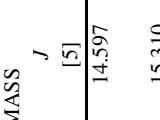

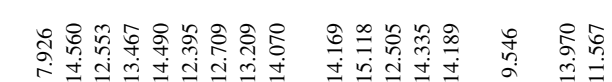

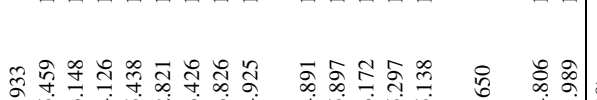

ind

(1)

旁

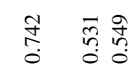

\section{辛}

疍

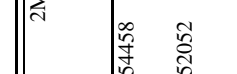

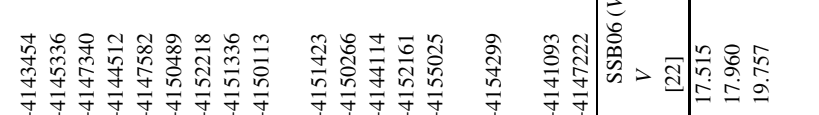

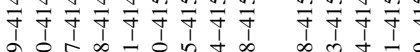

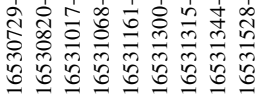

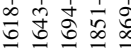

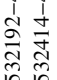

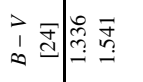

ㅇํㅇ

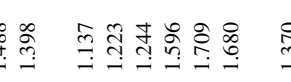

官

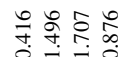

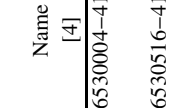

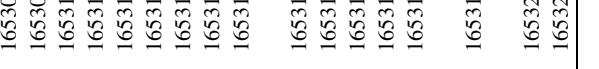

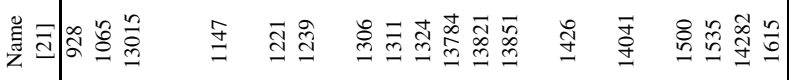

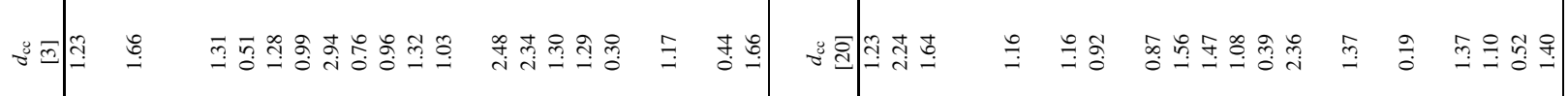
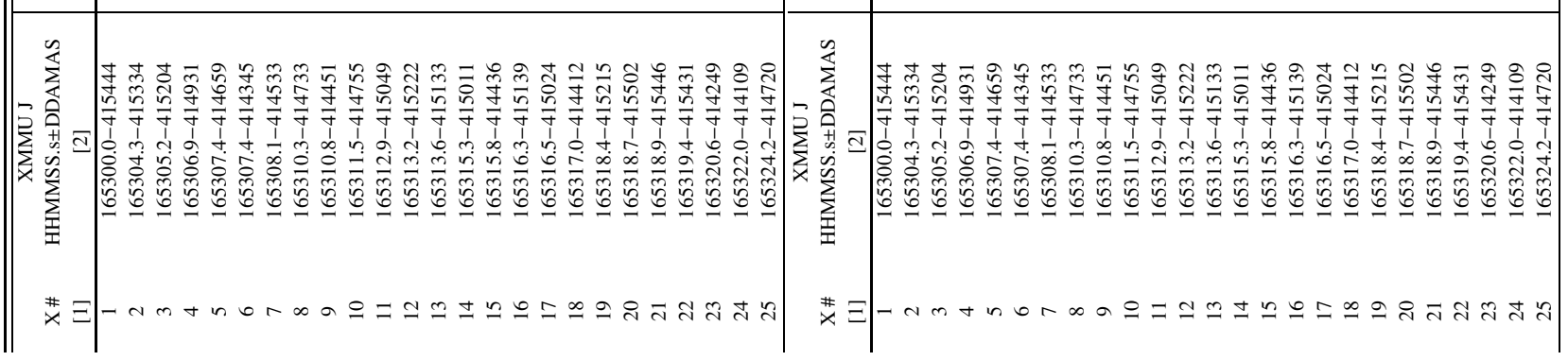
We adopted the following approach. Because of the presence of gaps, we computed an equivalent EPIC pn count rate, in the range $0.5-10.0 \mathrm{keV}$, for each source. To the first order, the relation between the count rates measured in any of the two MOS detectors and in the pn detector is approximately linear. Using the count rates obtained for sources that were detected on several EPIC instruments, we thus derived an empirical conversion factor between the MOS1, MOS2 and pn count rates. These factors were then used to obtain the so-called pn-equivalent count rates for all sources and, in particular, for those that fall in the gaps of one or several instruments. This yields approximately coherent count rates for the different sources, whatever their position on the detectors. Figure 5 displays the source pn-equivalent count rates as a function of the distance from the central axis of the FOV - assumed to be the position of the binary HD 152248. A lower limit is clearly seen in the distribution. Selecting the faintest sources (i.e. those displaying the lowest equivalent count rates) in successive rings centered on HD 152248 provides an approximate sampling of this limit. We then adjusted a polynomial and derived an empirical detection limit in terms of pn-equivalent count rates $\left(c r_{\mathrm{lim}}\right)$ as a function of the distance $(d)$ from the field axis. This limit (in units of $10^{-3} \mathrm{cnt} \mathrm{s}^{-1}$ ) is described by the following relation:

$$
\begin{aligned}
c r_{\lim }(d)= & 2.49214-0.65577 d+0.11822 d^{2} \\
& -0.00929 d^{3}+0.00030 d^{4}
\end{aligned}
$$

where $d$ is the distance to HD 152248 expressed in arcmin. Equation (5) is shown in Fig. 5. Clearly the detection limit is higher in the central part of the field $\left(d<5^{\prime}\right)$, most probably because of the higher source density and because bright sources are preferentially located in the inner part of the FOV. The sensitivity also decreases towards the CCD edges, as indicated both by the exposure maps and the sensitivity maps. Finally we used single temperature optically thin thermal plasma models of the Raymond-Smith type to convert the pn-equivalent count rates given by Eq. (5) to fluxes and luminosities. For this purpose, we adopted the conversion computed by the WebPIMMS converter $^{3}$, assuming a column density of $0.26 \times 10^{22} \mathrm{~cm}^{-2}$, typical of the interstellar absorbing column for the cluster. Results are displayed in Fig. 6 for three different plasma temperatures. In conclusion, the flux detection limit is approximately located between $3 \times 10^{-15}$ and $1.5 \times 10^{-14} \mathrm{erg} \mathrm{cm}^{-2} \mathrm{~s}^{-1}$, depending on the distance from the detector axis and on the source spectrum. In the central part of the FOV, we consider that the typical limiting flux is about $6 \times 10^{-15} \mathrm{erg} \mathrm{cm}^{-2} \mathrm{~s}^{-1}$ for soft sources.

\section{The X-ray sources in NGC 6231}

This section presents an overview of the main properties of the X-ray sources in NGC 6231. No attempt will be made here to investigate the characteristics of the different sub-populations of the cluster. This work is postponed to future devoted papers.

\subsection{Spatial distribution}

As seen from Figs. 1 to 3, there is an obvious clustering of the $\mathrm{X}$-ray sources in the inner part of the FOV. Their spatial distribution projected on the sky presents, at first sight, a revolution symmetry around the centre of the field, i.e. the position

\footnotetext{
${ }^{3}$ WebPIMMS is a NASA's HEASARC tool powered by PIMMS v3.6a. It is hosted at the following URL: http://heasarc.gsfc .nasa.gov/Tools/w3pimms.html
}

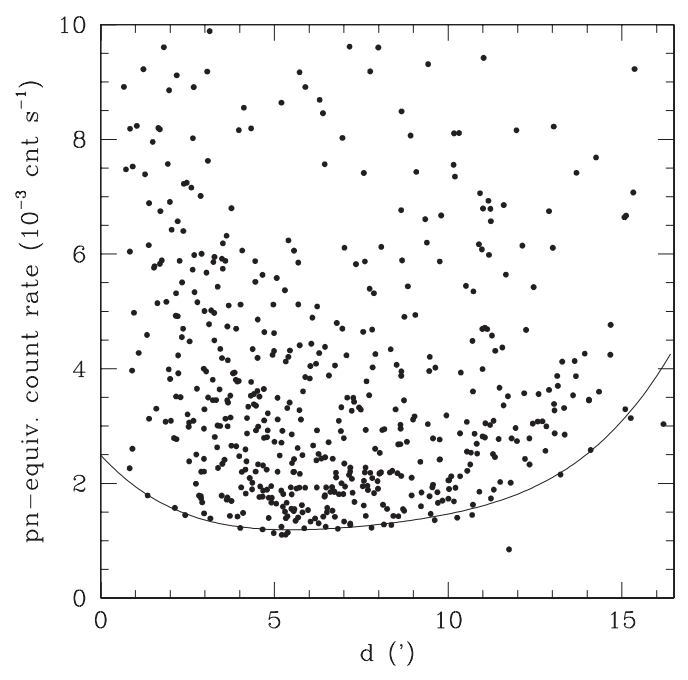

Fig. 5. Bottom part of the distribution of the pn-equivalent count rates of the X-ray sources as a function of their distance $(d)$ to HD 152248. The plain line shows the adopted lower limit given by Eq. (5).

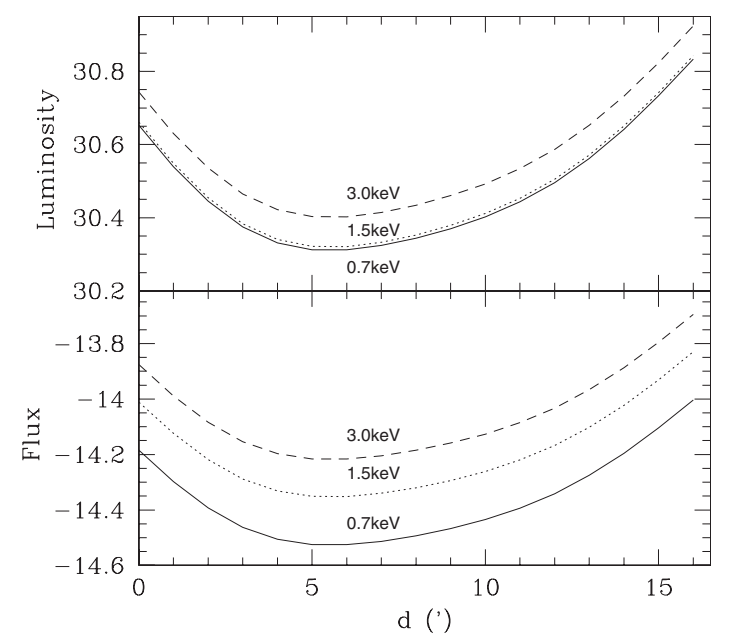

Fig. 6. Lower panel: estimated detection limit expressed in terms of the observed flux (in $\log \left(\operatorname{erg~} \mathrm{cm}^{-2} \mathrm{~s}^{-1}\right)$ ). Upper panel: equivalent detection limit, expressed in ISM-absorption corrected luminosity (in $\left.\log \left(\mathrm{erg} \mathrm{s}^{-1}\right)\right)$, for sources located in the NGC 6231 cluster $(D M=$ $11.07, n_{\mathrm{H}, \mathrm{ISM}}=0.26 \times 10^{22} \mathrm{~cm}^{-2}$ ). The different lines refer to the different MEKAL model temperatures adopted for the conversion. The energy band considered in both panels is $0.5-10.0 \mathrm{keV}$.

of HD 152248. Considering the sources located at less than $15^{\prime}$ from HD 152248, we computed the geometrical centre of the source distribution. We also computed the brightness centre of the X-ray image. For this purpose, we adopted the pn-equivalent count rates for each source. The two centres are located slightly East of HD 152248, at no more than 30" (see Fig. 3). From the two-dimensional map of the X-ray source density (Fig. 7), we conclude that there is only a slight deviation from this scheme and that the X-ray source distribution shows a slight N-S elongation. In the following, we however assume that the distance from the cluster centre, i.e. from HD 152248, remains the main parameter that shapes the source distribution. We also adopt the position of HD 152248 as the very centre of the cluster.

From Figs. 7 and 8, it is clear that the radial distribution of the sources is not uniform and that most of them lie within a $10^{\prime}$ radius around the cluster centre. We computed the radial density profile of the X-ray emitters and we adjusted an 


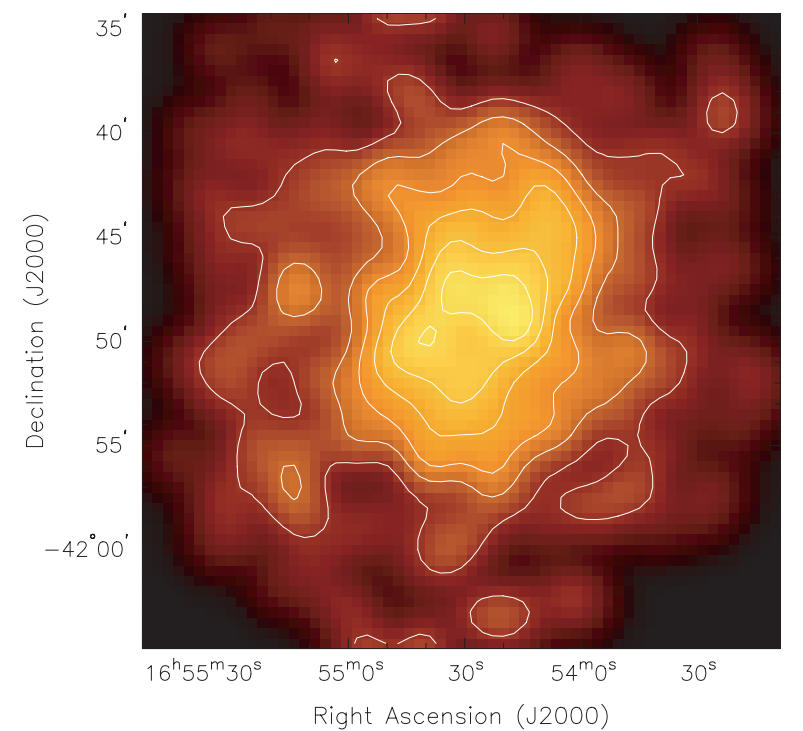

Fig. 7. 2D distribution of the surface density of the X-ray sources. The image is centered on the location of HD 152248. The original source density chart was convolved with a Gaussian with $\sigma=1^{\prime}$. Overplotted contour levels correspond to 1, 1.5, 2, 2.5, 3 and 3.5 sources per $\operatorname{arcmin}^{2}$.

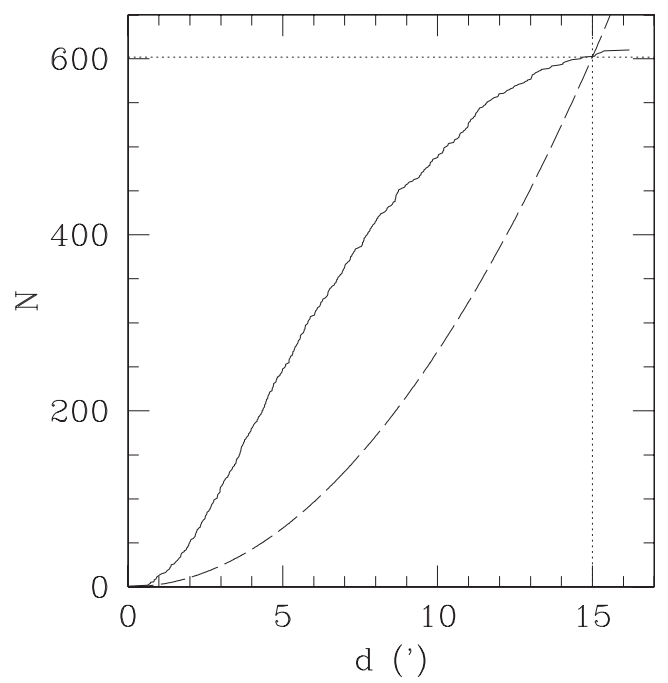

Fig. 8. Plain line: cumulative distribution of the number of X-ray sources $(N)$ with increasing distance $(d)$ from HD 152248. Dashed line: idem, but computed assuming a uniform distribution of the 602 sources (dotted horizontal line) located within a 15' (dotted vertical line) circle around HD 152248.

empirical King density profile (King 1962) for a spherically distributed source population:

$f(d)=k\left[1 / \sqrt{1+\left(d / d_{\mathrm{c}}\right)^{2}}-1 / \sqrt{1+\left(d_{\mathrm{t}} / d_{\mathrm{c}}\right)^{2}}\right]^{2}$

where $k$ is the central density, $d_{\mathrm{c}}$ the core radius and $d_{\mathrm{t}}$ the limiting radius. The King profile is very sensitive to $k$ and $d_{\mathrm{c}}$, but less sensitive to $d_{\mathrm{t}}$ which is indeed less meaningful for open clusters in the Galactic plane. The best fit parameters are $k=8.9 \operatorname{arcmin}^{-2}, d_{\mathrm{c}}=6.5$ and $d_{\mathrm{t}}=20.5$. As indicated by Figs. 5 and 6 , our detection limit depends on the location of the source on the detector. In a second step, we thus applied a relative correction to the $\mathrm{X}$-ray density profile, accounting for the sensitivity difference as a function of the distance to the

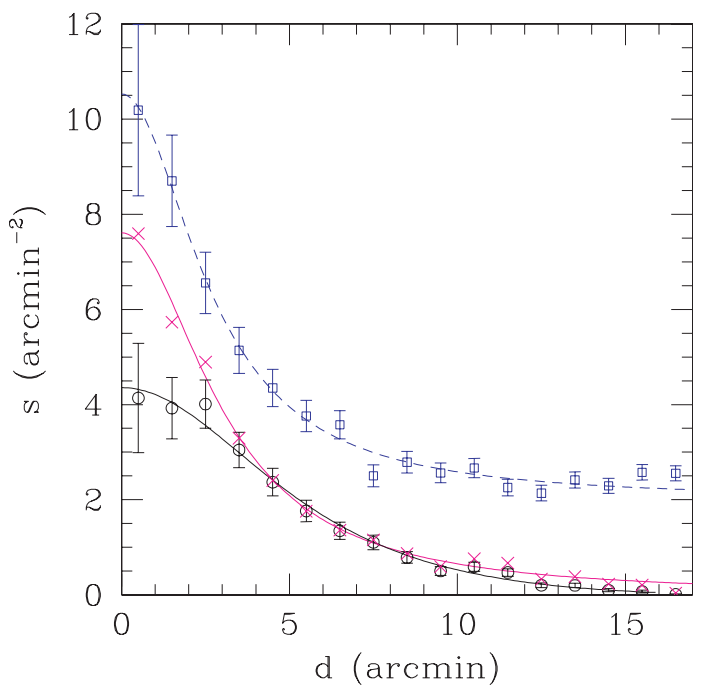

Fig. 9. Surface density profiles of the X-ray sources (open circles) and of the optical sources (open squares) with $V<17$. Crosses indicate the $\mathrm{X}$-ray density profile corrected for the empirical EPIC sensitivity curve (see text). Best-fit King profiles are overplotted.

detector axis (crosses in Fig. 9). The profile is now sharper and is described by: $k=7.6 \mathrm{arcmin}^{-2}, d_{\mathrm{c}}=3$. 1 and $d_{\mathrm{t}}=1.5 \times$ $10^{3}$ arcmin. In Fig. 9, we also present the density profile of the stars in SSB06. Investigating the source density distribution as a function of the limiting magnitude of the catalogue and of the distance to the detector axis, we further note that the SSB06 catalogue is almost undoubtedly incomplete in the field centre above $V=18$. This is easily explained by the number of bright sources $(V \approx 5-10$, see Fig. 3 ) in this region, that renders the detection of faint sources more difficult. For this reason, Fig. 9 is restricted to objects brighter than 17 in the $V$ band. NGC 6231 is further embedded in the Sco OB 1 association. As a consequence, the surface density does not drop to zero in the outer region of the field. We thus subtracted a threshold of $2 \mathrm{arcmin}^{-2}$ prior to the adjustment. King best-fit values are this time $k=8.6 \mathrm{arcmin}^{-2}$, $d_{\mathrm{c}}=2 ! 7$ and $d_{\mathrm{t}}=1.4 \times 10^{3}$ arcmin. From Fig. 9, the correlation between the $\mathrm{X}$-ray and optical surface density profile is obvious and yields similar core radii for NGC 6231. It further suggests that most of the detected X-ray emitters are physically belonging to NGC 6231.

As discussed in e.g. Sung et al. (2004), X-ray emission is probably one of the best membership criterion for young stars in open clusters. The present X-ray observations probably provide the best census so far of PMS stars in NGC 6231; though this census is probably still incomplete. However, the NGC 6231 X-ray sample might be contaminated by foreground (field stars) and background (AGNs) objects. As a last check, we thus roughly estimated the probable number of foreground and background X-ray sources detected in the present campaign. Starting with the foreground objects, we proceeded as explained below. Accounting for the different typical X-ray luminosities for field stars of spectral type $\mathrm{O}$ to $\mathrm{M}$ and for our flux detection limit, we estimated the maximum distance at which a star can be located while still being detected. Using the soderived distance, we computed the volume projected onto the XMM-Newton FOV. As a last step, we adopted typical star densities in the solar neighbourhood as quoted by Allen (1973) for the different spectral types. We finally end up with about 20 foreground X-ray sources, most of which are expected to be G-type objects (12 stars) and F-type dwarves (4 or 5 stars). However, 
the previous approach does not account for probable active stars or RS CVn in the FOV, which have lower spatial densities but much higher luminosities. Using the work of Makarov (2003b), we found that about 21 galactic active stars could be detected in the EPIC FOV. This yields a total of approximatively 41 contaminating galactic sources. As an additional check, we also used the X-ray stellar $\log N(>S)-\log S$ curve at low galactic latitudes provided by Motch et al. (2003). Again we found that about 40 galactic X-ray sources are to be expected within our EPIC FOV.

We then obtained a rough estimate of the number of extragalactic background sources in our EPIC field. The Galactic coordinates of the cluster are $l_{\mathrm{II}}=343.46, b_{\mathrm{II}}=+1$. 19 . Therefore, the total neutral hydrogen column density along this direction must be extremely large and should produce a substantial absorption of X-ray photons from extragalactic sources. Although they are in principle not suited for lines of sight at $\left|b_{\mathrm{II}}\right| \leq 5^{\circ}$, we used the DIRBE/IRAS extinction maps provided by Schlegel et al. (1998) to derive a first order estimate of the total column density. In this way, we find a total Galactic $E(B-V)$ of about 5.6 mag. Using the gas to dust ratio of Bohlin et al. (1978), we thus estimate a neutral hydrogen column density of $\sim 3.2 \times$ $10^{22} \mathrm{~cm}^{-2}$. Assuming that extragalactic background sources have a power-law spectrum with a photon index of 1.4, and suffer a total interstellar absorption of $3.2 \times 10^{22} \mathrm{~cm}^{-2}$, the mean detection limit $1.9 \times 10^{-3} \mathrm{cnt} \mathrm{s}^{-1}$ with the pn camera translates into unabsorbed fluxes of $1.2 \times 10^{-14}$ and $3.5 \times 10^{-13} \mathrm{erg} \mathrm{cm}^{-2} \mathrm{~s}^{-1}$ in the $0.5-2.0 \mathrm{keV}$ and $2.0-10 \mathrm{keV}$ band respectively. Using the $\log N-\log S$ relation from Giacconi et al. (2001), one expects thus about 13-16 extragalactic objects among the detected sources. Thus, about $2 \%$ of the total number of sources could be background AGNs. It should be emphasized that these background AGNs are expected to appear as rather hard (i.e. heavily absorbed) X-ray sources.

In summary, both the geometrical and X-ray brightness centres of the set of detected sources correspond to the optical cluster centre. The radial profile of the X-ray source density is well correlated with the optical source radial profile. Both indicate a cluster core radius close to $3^{\prime}$. Finally, we expect that less than $10 \%$ of the presently detected sources correspond to foreground or background objects. We thus propose that the large majority of the X-ray emitters revealed by the present XMM-Newton campaign are mostly belonging to NGC 6231. Some of them might alternatively belong to the Sco OB 1 association, in which NGC 6231 is embedded.

\subsection{Emission distribution}

While HD 152248, the brightest X-ray emitter in the FOV, displays a pn-equivalent count rate larger than $0.36 \mathrm{cnt} \mathrm{s}^{-1}$, most of the other sources are much fainter with a count rate below $10^{-2} \mathrm{cnt} \mathrm{s}^{-1}$ (Fig. 10). It is clear from Fig. 1 that most of the brightest sources - associated with the O-type objects of the cluster - are relatively soft while the majority of the $\mathrm{X}$-ray emitters have their maximum of emission in the medium band. Except for the brightest sources, there is no obvious correlation between the source intensity and the source hardness ratios. On average, the detected sources are moderately hard with $H R_{1}>0$ and $H R_{2}<0$ (Fig. 11). The hardness ratios might however show a slight increase towards the edge of the detectors, probably due to the relative dominance of lowmass stars in the outer regions of the FOV. The histograms of the detected source count rates in the $S_{\mathrm{X}}, M_{\mathrm{X}}$ and $H_{\mathrm{X}}$ bands (Fig. 12) reveal clear peaks around 0.7, 1.0 and $0.2 \times 10^{-3} \mathrm{cnt} \mathrm{s}^{-1}$

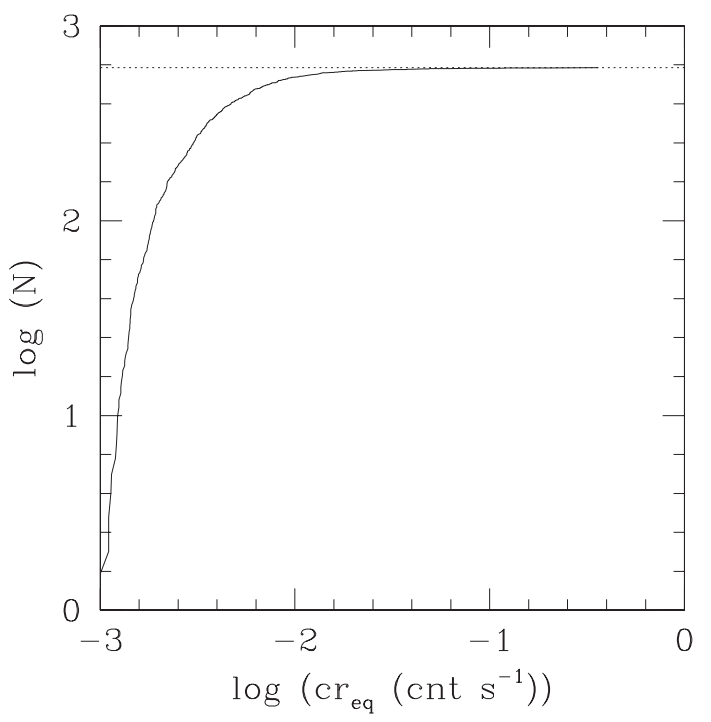

Fig. 10. Cumulative distribution of the number of sources as a function of increasing pn-equivalent count rate. The horizontal dotted line indicates the total number of sources in the FOV.

respectively. The count rate in the $0.5-10.0 \mathrm{keV}$ band clusters at $2 \times 10^{-3} \mathrm{cnt} \mathrm{s}^{-1}$ and the two hardness ratios around 0.2 and -0.6 respectively. Accounting for the cluster typical ISM absorbing column $n_{\mathrm{H}, \mathrm{ISM}}=0.26 \times 10^{22} \mathrm{~cm}^{-2}$, these values are roughly described by a MEKAL model with a temperature of $k T=1.0-2.0 \mathrm{keV}$. The corresponding observed flux is about $5 \times$ $10^{-15} \mathrm{erg} \mathrm{cm}^{-2} \mathrm{~s}^{-1}$. Adopting a distance modulus $D M=11.07$, this yields a $\operatorname{luminosity~} \log \left(L_{\mathrm{X}}\right) \sim 30.5\left(\mathrm{erg} \mathrm{s}^{-1}\right)$ for a typical $\mathrm{X}$-ray emitter in the cluster.

\section{Summary}

We presented the first results of an XMM-Newton campaign on the young open cluster NGC 6231 in the Sco OB 1 association. With an effective cumulated exposure time of $175 \mathrm{ks}$ in the two EPIC MOS instruments and of $147.5 \mathrm{ks}$ in the EPIC pn, the campaign was split into six successive observations acquired within 5 days. The combined image, built from the data collected by the three EPIC instruments during the six pointings, reveals an extremely crowded field. Running the SAS task emldetect, we detect 610 X-ray sources among which 19 are reported as extended. The latter are probably constituted by non-resolved point-like sources rather than by physically extended sources. We present an X-ray catalogue covering the XMM-Newton FOV and we cross-correlate it with several optical/infrared catalogues. We find at least one optical and/or infrared counterpart for more than $85 \%$ of the X-ray sources within a limited cross-correlation radius of $3^{\prime \prime}$ at maximum. We estimate our detection flux limit to lie between about $3 \times 10^{-15}$ and $1.5 \times$ $10^{-14} \mathrm{erg} \mathrm{cm}^{-2} \mathrm{~s}^{-1}$ depending on the position on the detectors and on the source spectrum.

The surface density distribution of the X-ray sources peaks at the centre of the cluster, which we find to be located very near HD 152248, and presents a slight N-S elongation. Concerning the radial profile of the surface density distribution, over $50 \%$ of the sources are confined within a $6^{\prime}$ radius from the cluster centre and about $80 \%$ within $10^{\prime}$. The estimated contamination by foreground and background objects is about $9 \%$. There is a good similarity between this radial profile and the distribution of stars brighter than $V=17$, suggesting that most of the sources 

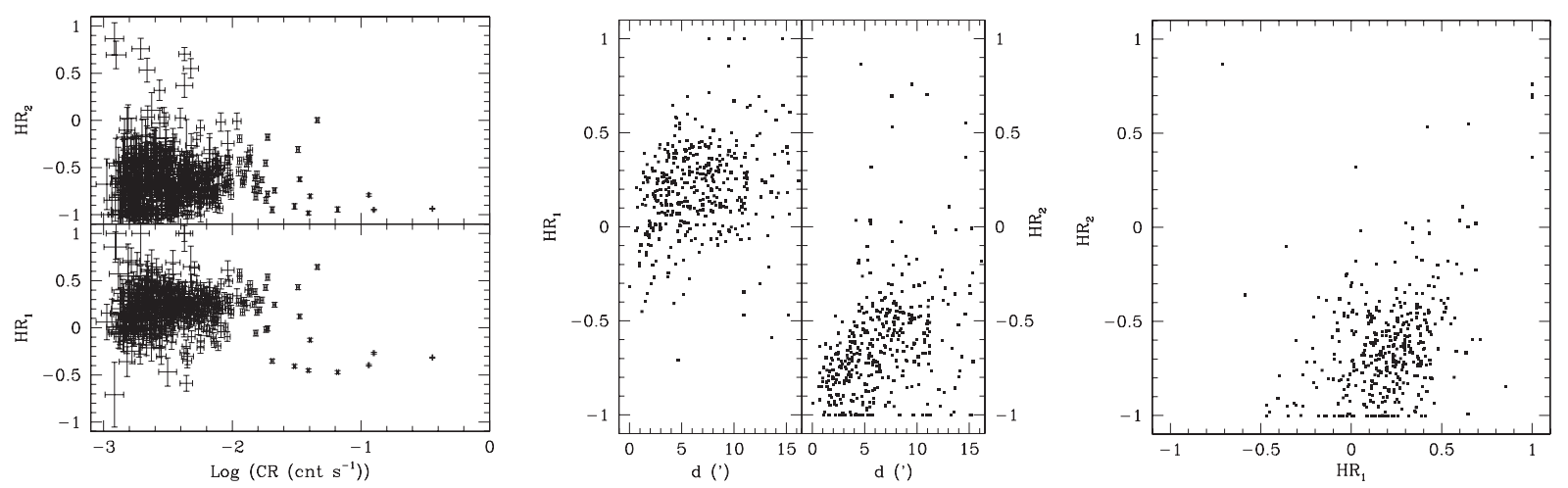

Fig. 11. Left panel: hardness ratios versus count rate. Middle panel: hardness ratios versus distance $(d)$ from the FOV centre. Right panel: $H R_{2}$ versus $H R_{1}$. The three panels are built for the sources detected with the pn instrument. Results for the EPIC MOS instruments are similar.

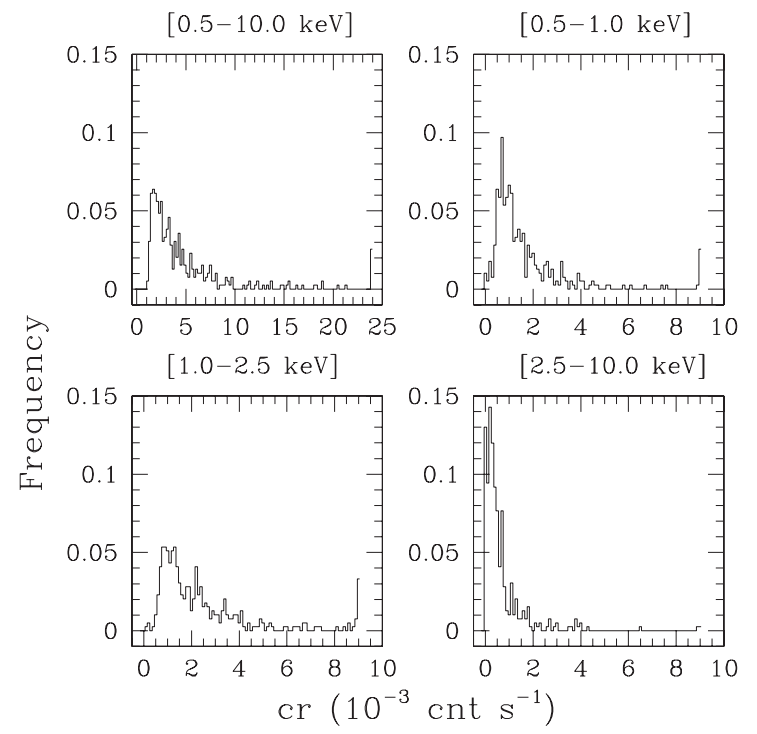

Fig. 12. Distribution of the X-ray emitter count rates in the different energy bands considered. The four panels were plotted only using the 392 sources detected with the pn instrument. Results for the EPIC MOS instruments are similar. The last bin includes the contributions of all the brightest X-ray sources.

physically belong to NGC 6231. The radial surface density profile of the X-ray sources is well described by a King profile with a core radius of about $3^{\prime}$, similar to the one indicated by the $V<17$ optical source density profile.

Finally, beside a few bright and soft objects correlated with the O-type stars of the cluster, the large majority of the X-ray population is relatively faint (pn-equivalent count rate below $10^{-2} \mathrm{cnt} \mathrm{s}^{-1}$ ) and displays an intermediate spectrum of a typical temperature probably around 1.0-2.0 keV. Typical count rates for the sources are 2.0, 0.7, 1.0 and $0.2 \times 10^{-3} \mathrm{cnt} \mathrm{s}^{-1}$ respectively in the total energy band $(0.5-10.0 \mathrm{keV})$, and in the three energy sub-ranges $0.5-1.0,1.0-2.5$ and $2.5-10.0 \mathrm{keV}$. At the NGC 6231 cluster distance, these values roughly correspond to an X-ray luminosity of about $\log \left(L_{\mathrm{X}}\right) \sim 30.5\left(\mathrm{erg} \mathrm{s}^{-1}\right)$.

More detailed investigations of the X-ray properties of the different sub-populations (early-type stars, PMS objects) of the cluster will be presented in subsequent papers in this series. Finally, the X-ray data related to specific early-type binary systems of particular interest are (will be) presented in dedicated papers (see e.g. Sana et al. 2004, 2005a, 2006a; as well as Sana 2005), together with the derivation of their orbital and physical parameters obtained on the basis of an extensive spectral monitoring campaign in the optical domain.

Acknowledgements. It is a pleasure to thank Dr. W. Seggewiss for providing a copy of his original paper on the cluster photometry (Seggewiss 1968b), our system manager, A. Detal, for essential assistance in installing and handling the successive SAS versions and Dr. J.-F. Claeskens for sharing numerical routines. We are also grateful to the referee, Dr. R. Jeffries, for comments and suggestions that helped to improve the overall quality of the paper. This research is supported in part by contract P5/36 "Pôle d'Attraction Interuniversitaire" (Belgian Federal Science Policy Office) and through the PRODEX XMM and INTEGRAL contracts. H. Sung acknowledges the support of the Korea Science and Engineering Foundation (KOSEF) to the Astrophysical Research Center for the Structure and Evolution of the Cosmos (ARCSEC ${ }^{\prime \prime}$ ) at Sejong University.

This publication makes use of data products from USNO B1.0, the Two Micron All Sky Survey (2MASS) and the Guide Star catalogue (GSC) II. The 2MASS is a joint project of the University of Massachusetts and the Infrared Processing and Analysis Center/California Institute of Technology, funded by the NASA and the NSF. The GSC II is a joint project of the Space Telescope Science Institute and the Osservatorio Astronomico di Torino. Additional support is provided by the ESO, the Space Telescope European Coordinating Facility, the International GEMINI project and the ESA Astrophysics Division. The SIMBAD database and the Vizier catalogue access tool (CDS, Strasbourg, France) have been consulted for the bibliography and for the purpose of object cross-identification.

\section{References}

Allen, C. W. 1973, Astrophysical quantities (London: University of London, Athlone Press), 3rd edn.

Arenou, F., \& Luri, X. 1999, in Harmonizing Cosmic Distance Scales in a PostHIPPARCOS Era, ed. D. Egret, \& A. Heck, ASP Conf. Ser., 167, 13

Arentoft, T., Sterken, C., Knudsen, M. R., et al. 2001, A\&A, 380, 599

Balona, L. A. 1983, MNRAS, 203, 1041

Balona, L. A. 1992, MNRAS, 254, 404

Balona, L. A., \& Engelbrecht, C. A. 1985, MNRAS, 212, 889

Balona, L. A., \& Laney, C. D. 1995, MNRAS, 276, 627

Baume, G., Vázquez, R. A., \& Feinstein, A. 1999, A\&AS, 137, 233

Bohlin, R. C., Savage, B. D., \& Drake, J. F. 1978, ApJ, 224, 132

Bok, B. J., Bok, P. F., \& Graham, J. A. 1966, MNRAS, 131, 247

Braes, L. L. E. 1967, Bull. Astron. Inst. Netherl. Suppl. Ser., 2, 1

Breckinridge, J. B., \& Kron, G. E. 1963, PASP, 75, 248

Brownlee, R. R., \& Cox, A. N. 1953, ApJ, 118, 165

Cash, W. 1979, ApJ, 228, 939

Conti, P. S., \& Alschuler, W. R. 1971, ApJ, 170, 325

Corcoran, M. F. 1996, Rev. Mex. Astron. Astrofis. Conf. Ser., 5, 54

Corcoran, M. F. 1999, Rev. Mex. Astron. Astrofis. Conf. Ser., 8, 131

Crawford, I. A. 2001, MNRAS, 328, 1115

Crawford, D. L., Barnes, J. V., Hill, G., \& Perry, C. L. 1971, AJ, 76, 1048

Cutri, R. M., Skrutskie, M. F., van Dyk, S., et al. 2003, University of Massachusetts and Infrared Processing and Analysis Center (IPAC/California Institute of Technology)

Damiani, F., Flaccomio, E., Micela, G., et al. 2004, ApJ, 608, 781

den Herder, J. W., Brinkman, A. C., Kahn, S. M., et al. 2001, A\&A, 365, L7

Feast, M. W., Stoy, R. H., Thackeray, A. D., \& Wesselink, A. J. 1961, MNRAS, 122, 239

Feinstein, A., \& Ferrer, O. E. 1968, PASP, 80, 410 
Feinstein, C., Martínez, R., Vergne, M. M., Baume, G., \& Vázquez, R. 2003, ApJ, 598, 349

García, B., \& Mermilliod, J. C. 2001, A\&A, 368, 122

Garrison, R. F., \& Schild, R. E. 1979, AJ, 84, 1020

Giacconi, R., Rosati, P., Tozzi, P., et al. 2001, ApJ, 551, 624

GSC 2.2. 2001, Space Telescope Science Institute (STScI) and Osservatorio Astronomico di Torino

Heske, A., \& Wendker, H. J. 1984, A\&AS, 57, 205

Hill, G., Crawford, D. L., \& Barnes, J. V. 1974, AJ, 79, 1271

Houck, T. E. 1956, Ph.D. Thesis

Jansen, F., Lumb, D., Altieri, B., et al. 2001, A\&A, 365, L1

Jeffries, R. D., Thurston, M. R., \& Pye, J. P. 1997, MNRAS, 287, 350

King, I. 1962, AJ, 67, 471

Laval, A. 1972, A\&A, 21, 271

Levato, H., \& Malaroda, S. 1980, PASP, 92, 323

Levato, H., \& Morrell, N. 1983, Astrophys. Lett., 23, 183

Levato, H., Morrell, N., Garcia, B., \& Malaroda, S. 1988, ApJS, 68, 319

Lührs, S. 1997, PASP, 109, 504

Makarov, V. V. 2003a, AJ, 126, 2408

Makarov, V. V. 2003b, AJ, 126, 1996

Marggraf, O., Bluhm, H., \& de Boer, K. S. 2004, A\&A, 416, 251

Mason, B. D., Gies, D. R., Hartkopf, W. I., et al. 1998, AJ, 115, 821

Mason, K. O., Breeveld, A., Much, R., et al. 2001, A\&A, 365, L36

Mathys, G. 1988, A\&AS, 76, 427

Mathys, G. 1989, A\&AS, 81, 237

Mermilliod, J. C. 1981, A\&A, 97, 235

Meynet, G., Mermilliod, J.-C., \& Maeder, A. 1993, A\&AS, 98, 477

Monet, D. G., Levine, S. E., Canzian, B., et al. 2003, AJ, 125, 984

Morgan, W. W., González, G., \& González, G. 1953a, ApJ, 118, 323

Morgan, W. W., Whitford, A. E., \& Code, A. D. 1953b, ApJ, 118, 318

Motch, C., Herent, O., \& Guillout, P. 2003, Astron. Nachr., 324, 61

Penny, L. R., Bagnuolo, W. G., \& Gies, D. R. 1994, Space Sci. Rev., 66, 323

Penny, L. R., Gies, D. R., \& Bagnuolo, W. G. 1999, ApJ, 518, 450

Perry, C. L., Hill, G., Younger, P. F., \& Barnes, J. V. 1990, A\&AS, 86, 415

Perry, C. L., Hill, G., \& Christodoulou, D. M. 1991, A\&AS, 90, 195

Preibisch, T., \& Zinnecker, H. 2004, A\&A, 422, 1001

Raboud, D. 1996, A\&A, 315, 384

Raboud, D., \& Mermilliod, J.-C. 1998, A\&A, 333, 897

Raboud, D., Cramer, N., \& Bernasconi, P. A. 1997, A\&A, 325, 167

Rauw, G., Nazé, Y., Gosset, E., et al. 2002, A\&A, 395, 499

Rauw, G., De Becker, M., Gosset, E., Pittard, J. M., \& Stevens, I. R. 2003, A\&A, 407,925
Sana, H. 2005, Ph.D. Thesis, Liège University

Sana, H., Rauw, G., \& Gosset, E. 2001, A\&A, 370, 121

Sana, H., Rauw, G., Gosset, E., \& Vreux, J.-M. 2002, in Interacting Winds from Massive Stars, ed. A. Moffat, \& N. St.-Louis, ASP Conf. Ser., 260, 431

Sana, H., Hensberge, H., Rauw, G., \& Gosset, E. 2003, A\&A, 405, 1063

Sana, H., Stevens, I. R., Gosset, E., Rauw, G., \& Vreux, J.-M. 2004, MNRAS, 350,809

Sana, H., Antokhina, E., Royer, P., et al. 2005a, A\&A, 441, 213

Sana, H., Rauw, G., \& Gosset, E. 2005b, in Massive Stars and High-Energy Emission in OB Associations, Proc. JENAM 2005 Distant Worlds, ed. G. Rauw et al., 107

Sana, H., Gosset, E., \& Rauw, G. 2006a, MNRAS, in press

Sana, H., Gosset, E., Rauw, G., \& Vreux, J.-M. 2006b, in the X-Ray Universe 2005, ed. A. Wilson, ESA SP-604, 21

Sana, H., Nazé, Y., Gosset, E., et al. 2006c, in Massive Stars in Interacting Binaries, ed. A. Moffat, \& N. St-Louis, ASP Conf. Ser., 5, in press

Schild, R. E., Hiltner, W. A., \& Sanduleak, N. 1969, ApJ, 156, 609

Schlegel, D. J., Finkbeiner, D. P., \& Davis, M. 1998, ApJ, 500, 525

Seggewiss, W. 1968a, Z. Astrophys., 68, 142

Seggewiss, W. 1968b, Veroeffentlichungen des Astronomischen Institut der Universitaet Bonn, 79

Setia Gunawan, D. Y. A., Chapman, J. M., Stevens, I. R., Rauw, G., \& Leitherer, C. 2002, private communication

Setia Gunawan, D. Y. A., Chapman, J. M., Stevens, I. R., Rauw, G., \& Leitherer, C. 2003, in A Massive Star Odyssey: from main sequence to supernova, ed. K. van der Hucht, A. Herrero, \& C. Esteban, IAU Symp., 212, 230

Shobbrook, R. R. 1983, MNRAS, 205, 1229

Skinner, S., Gagné, M., \& Belzer, E. 2003, ApJ, 598, 375

Stickland, D. J., \& Lloyd, C. 2001, The Observatory, 121, 1

Stickland, D. J., Lloyd, C., Penny, L. R., Gies, D. R., \& Bagnuolo, W. G. 1996, The Observatory, 116, 226

Stickland, D. J., Lloyd, C., \& Penny, L. R. 1997, The Observatory, 117, 213

Strüder, L., Briel, U., Dennerl, K., et al. 2001, A\&A, 365, L18

Struve, O. 1944, ApJ, 100, 189

Sung, H., Bessell, M. S., \& Lee, S. 1998, AJ, 115, 734

Sung, H., Bessell, M. S., \& Chun, M. 2004, AJ, 128, 1684

Turner, M. J. L., Abbey, A., Arnaud, M., et al. 2001, A\&A, 365, L27

van Genderen, A. M., Bijleveld, W., \& van Groningen, E. 1984, A\&AS, 58, 537

Walborn, N. R. 1972, AJ, 77, 312

Walraven, T., \& Walraven, J. H. 1960, Bull. Astron. Inst. Netherl., 15, 67 


\section{Online Material}




\section{Appendix A: On correcting the $L_{2}$ values in SAS v 5.4.1}

The equivalent (or transformed) logarithmic likelihood $L_{2}$ associated with each source candidate detected by the SAS task emldetect (column DET_ML in the output file) is given by:

$L_{2}=-\ln \left(1-P\left(\frac{v}{2}, L^{\prime}\right)\right)$

with

$L^{\prime}=\sum_{i=1}^{i=n} l_{i}$

where $P$ is the incomplete Gamma function, $v$ is the number of degrees of freedom (d.o.f.) of the fit, $n$ is the number of input images (i.e. the number of energy bands times the number of instruments considered), and $l_{i}=C_{i} / 2$ with $C_{i}$ being the Cash statistic for image $i$, specially designed by Cash (1979) for photon counting experiments. More insight into the physical meaning of Eq. (A.1) will be given in the next section. In this section we focus on the implemented patch for correcting $L_{2}$ values.

Indeed the logarithmic likelihood $L_{2}$ is known to be erroneous in SAS version v5.4.1 and earlier versions (XMM-Newton News \#29 - 11-Mar.-2003). According to SAS Observation Report SASv5.4/8665 ${ }^{4}$, the factor 2 in equation $l_{i}=C_{i} / 2$ has been forgotten, leading to erroneous $L^{\prime}$ and hence $L_{2}$. Knowing the number of degrees of freedom $v$, it is a simple exercise to invert Eq. (A.1) and to obtain values for $L^{\prime}$. From Eq. (A.2), it is obvious that the corrected value for $L^{\prime}$ is $L_{\text {corr }}^{\prime}=L^{\prime} / 2$, to be used in Eq. (A.1) to recover the corrected $L_{2}^{\text {corr }}$ value that can then be used for scientific analyses.

For large values of $L_{2}\left(L_{2} \gtrsim 10000\right)$ the numerical limits of classical compilers are however exceeded. Fortunately Eq. (A.1) tends to a linear relation between $L^{\prime}$ and $L_{2}$ for large values and for a given $v$. The correction is therefore straightforward with $L_{2}^{\text {corr }}=L_{2} / 2$. Though this bug was present at the time we analysed the data, this issue has been fixed later in SAS version v 6.0. We checked our corrected $L_{2}^{\text {corr }}$ values against SAS v 6.0 and found them in close agreement.

\section{Appendix B: On the choice of coherent detection thresholds using the transformed logarithmic likelihood $L_{2}$}

As it can be deduced from the previous section (App. A), the logarithmic likelihood $L_{2}$ is related to the probability that a detected source candidate could be explained by pure random Poissonian fluctuations (and zero count in the source). Computed for each source of the input list, it uses a combination of the Cash statistic $C_{i}$ obtained for the different input images $i$. The Cash statistic $C_{i}$ actually results from a likelihood ratio test and obeys a $\chi^{2}$ distribution (Cash 1979) with 3 or 4 degrees of freedom (i.e. the intensity, the $X$ - and $Y$-coordinates of the source and, eventually, the extent of the source if allowed). Therefore any linear combination of $n C_{i}$, and hence any computed $2 L^{\prime}$, also follows a $\chi^{2}$ statistic with $n+2$ or $n+3$ d.o.f. In this sense, the transformed logarithmic likelihood $L_{2}$ is indeed linked, through the simple relationship

$L_{2}=-\ln (Q)$,

\footnotetext{
${ }^{4}$ http://xmm.vilspa.esa.es/xmmhelp/
}

Table B.1. Illustration of consistently determined $L_{2}$ thresholds (Col. 2) for the different instruments and instrument combinations reported in Col. 1. The number of input images $(n)$ and corresponding degrees of freedom $(v)$ are given in Cols. 3 and $4 . L^{\prime}(\mathrm{Col} .5)$ is linked to $L_{2}$ through Eq. (A.1). A given $L^{\prime}$ is also linked to other $L^{\prime}$ of this table through Eq. (A.2) (see text). We emphasize that adopting any of the $L_{2}$ or $L^{\prime}$ presented in this table automatically determines the other values of $L^{\prime}$ and $L_{2}$ reported here below.

\begin{tabular}{ccccc}
\hline \hline Instr. Comb. & $L_{2}$ & $n$ & $v$ & $L^{\prime}$ \\
\hline MOS1 & 10.00 & 3 & 5 & 13.75 \\
MOS2 & 10.00 & 3 & 5 & 13.75 \\
pn & 22.77 & 3 & 5 & 27.51 \\
MOS1+MOS2 & 19.25 & 6 & 8 & 27.51 \\
MOS1+pn & 31.70 & 6 & 8 & 41.26 \\
MOS2+pn & 31.70 & 6 & 8 & 41.26 \\
MOS1+MOS2+pn & 40.86 & 9 & 11 & 55.02 \\
\hline
\end{tabular}

where

$Q=Q\left(\frac{\nu}{2}, L^{\prime}\right)=1-P\left(\frac{\nu}{2}, L^{\prime}\right)$

to the probability $Q$ for a random Poissonian fluctuation to have caused such a high value of $2 L^{\prime}=\sum_{i=1}^{i=n} C_{i}$ as the one observed. The equivalent logarithmic likelihood $L_{2}$ will therefore be large if the observed source is likely not produced by a statistical fluctuation, and small otherwise.

As a consequence, a threshold in $L_{2}$ can in principle be adopted as a detection limit. However, as we show below, while the expression given in Eq. (B.2) indeed takes into account the number $v$ of d.o.f. to compute the $Q$ probability and the subsequent value of $L_{2}$, it does not allow a direct comparison between $L_{2}$ obtained with different numbers of input images. This statement is illustrated in the following due consideration.

Let us assume that we are dealing, for example, with 3 energy bands and let us only consider point-like source fitting (parameter withextendedsource = "no"). For the purpose of the demonstration, let us adopt a uniform detection threshold, for any instrument or instrument combination, of $L_{2}=10$.

As a first step, let us deal with the source detection on the EPIC MOS1 images. In this particular configuration, there are three input images $(n=3)$ that correspond to the three energy bands. From the inversion of Eq. (A.1) with $L_{2}^{\mathrm{MOS} 1}=10$ and $v=$ 5 , we obtain $L_{\mathrm{MOS} 1}^{\prime}=13.75$, where $L_{\mathrm{MOS} 1}^{\prime}$ is the sum of the $l_{i}^{\mathrm{MOS} 1}$ for each of the three input images as given by Eq. (A.2), i.e.

$L_{\mathrm{MOS} 1}^{\prime}=\sum_{i=1}^{i=3} l_{i}^{\mathrm{MOS} 1}$

Now assuming that the two instruments MOS1 and MOS2 are exactly identical, a detection threshold $L_{2}^{\mathrm{MOS} 2}=10$ similarly corresponds to $L_{\mathrm{MOS} 2}^{\prime}=\sum_{i=1}^{i=3} l_{i}^{\mathrm{MOS} 2}=13.75$.

In a next step, let us work with a combination of the two EPIC MOS instruments. Equation (A.2) allows us to easily build the combined $L_{\mathrm{MOS} 1+\mathrm{MOS} 2}^{\prime}$ as the sum of the $l_{i}$ for each instrument and energy band:

$L_{\mathrm{MOS} 1+\operatorname{MOS} 2}^{\prime}=\sum_{i=1}^{i=3} l_{i}^{\mathrm{MOS} 1}+\sum_{i=1}^{i=3} l_{i}^{\mathrm{MOS} 2}=27.51$.

With two instruments and hence 6 images, $L_{\text {MOS1+MOS2 fol- }}^{\prime}$ lows a $\chi^{2}$ distribution with 8 d.o.f. $(v=8)$. Equation (A.1) then gives $L_{2}^{\mathrm{MOS} 1+\mathrm{MOS} 2}=19.25$ quite different from the value 
$L_{2}^{\mathrm{MOS} 1+\mathrm{MOS} 2}=10$ obtained with the adopted constant threshold limit $L_{2}=10$.

If we consider the use of two identical detectors, the fact, on one side, to combine them and, on the other side, to adopt the same statistical limit for both an isolated detector and a pair of them, allows us to go deeper. Actually, the combined logarithmic likelihood is twice the individual ones:

$L_{\mathrm{MOS} 1+\mathrm{MOS} 2}^{\prime}=2 L_{\mathrm{MOS} 1}^{\prime}=2 L_{\mathrm{MOS} 2}^{\prime}$.

Thus, this kind of threshold does not preserve the detection limit which is dependent on the particular combination used. If we want to preserve the detection limit adopted for a single instrument, we must, in this example, also multiply the detection threshold by a factor of two, adopting the value 27.51 instead of 13.75 and consequently 19.25 instead of 10 for the transformed $L_{2}$ statistic. We can of course extend this result to the pn detector. Making the reasonable assumption that $L_{\mathrm{pn}}^{\prime} \approx$ $2 L_{\mathrm{MOS}}^{\prime}$, a similar reasoning gives $L_{2}^{\mathrm{pn}}=22.77, L_{2}^{\mathrm{MOS} 1+\mathrm{pn}}=$ $L_{2}^{\mathrm{MOS} 2+\mathrm{pn}}=31.70$ and $L_{2}^{\mathrm{MOS} 1+\mathrm{MOS} 2+\mathrm{pn}}=40.86$, far from the value of 10.0 initially adopted. The intermediate results and numbers of d.o.f. used in establishing these values are given in Table B.1. Basically, when combining several instruments together, we improve the Poissonian statistics. The fact of adopting a constant value for $L_{2}$ for various instrumental combinations implies a cut-off in fluxes or count rates that is dependent on the number of detectors considered. Instead, if we prefer to stabilize the cut-off in absolute values of the signal rather independently of the combination used, we have to adapt the $L_{2}$ value to the situation.

In summary, one of the main results of the present discussion is that one should not adopt a constant threshold limit in $L_{2}$ for different instrument combinations if one wishes to preserve the uniformity of a cut-off level adopted for a given instrument or combination. We have shown that the $L_{2}$ thresholds in different combinations are linked through Eqs. (B.1) and (B.2) and through the detector physical characteristics that condition the $C_{i}$ values. In consequence, adopting a particular value as a threshold for a specific instrument or instrument combination implicitly assigns related values to the $L_{2}$ thresholds for any other instrument or combination considered. Therefore, if one wants to adopt a consistent detection threshold whatever the considered instrument or combination are, the previous reasoning becomes a forced step. This issue is particularly relevant to consistently deal with sources that fall on gaps or on specific detector areas where the different instruments do not overlap. We remind that this does not modify the spatial response of the detectors (nor the effect of the field crowdedness). Thus, spatial variations in the effective count rate threshold are still to be expected and, indeed, they are observed (see Figs. 5 and 6).

We finally remind the reader that the above presented method to determine self-consistent $L_{2}$ thresholds rests on two simplifying, but reasonable, assumptions. The first is that the two EPIC MOS instruments are identical. The second is that the EPIC pn yields approximately $L_{\mathrm{pn}}^{\prime} \approx 2 L_{\mathrm{MOS}}^{\prime}$. Any refinement of these two assumptions (i.e. any relation giving the $L^{\prime}$ of one instrument as a more realistic function of the $L^{\prime}$ of the other instruments) can be easily included in the method. This is however beyond the scope of the present discussion. The procedure illustrated here has been used in the making of our catalogue. The figures appearing in Table 2 were indeed established in a similar way (adopting $L_{2}^{\mathrm{MOS} 1}=L_{2}^{\mathrm{MOS} 2}=11$ ) and represent the threshold actually utilized. 